\title{
The Age and Metallicity Relation of Omega Centauri
}

\author{
Laura M. Stanford, Gary S. Da Costa and John E. Norris \\ Research School of Astronomy and Astrophysics, Australian National University, Weston, \\ ACT, 2611, Australia \\ stanford, gdc, jen@mso.anu.edu.au \\ and \\ Russell D. Cannon \\ Anglo-Australian Observatory, P.O. Box 296, Epping, NSW, 2121, Australia \\ rdc@aaoepp.gov.au
}

\begin{abstract}
We present a metallicity distribution based on photometry and spectra for $442 \omega$ Centauri cluster members that lie at the main sequence turnoff region of the color-magnitude diagram. This distribution is similar to that found for the red giant branch. The distribution shows a sharp rise to a mean of $[\mathrm{Fe} / \mathrm{H}]=-1.7$ with a long tail to higher metallicities. Ages have then been determined for the stars using theoretical isochrones enabling the construction of an age-metallicity diagram. Interpretation of this diagram is complicated by the correlation of the errors in the metallicities and ages. Nevertheless, after extensive MonteCarlo simulations, we conclude that our data show that the formation of the cluster took place over an extended period of time: the most metal-rich stars in our sample $([\mathrm{Fe} / \mathrm{H}] \approx-0.6)$ are younger by $2-4$ Gyrs than the most metal-poor population.
\end{abstract}

Subject headings: globular clusters: general — globular clusters: individual $(\omega$ Centauri)

\section{Introduction}

The Galactic globular cluster $\omega$ Centauri exhibits unusual properties compared to other clusters. The first indication that it was atypical was in the photometric work of Woolley et al. (1966) and Cannon \& Stobie (1973a) where the large color width of the red giant 
branch (RGB) was first established. An internal spread in metallicity was shown to exist from the spectroscopic work of Freeman \& Rodgers (1975) using RR Lyrae stars. A large range in metallicity from $[\mathrm{Fe} / \mathrm{H}]=-1.8$ up to $[\mathrm{Fe} / \mathrm{H}] \sim-0.4$, and several discrete populations on the RGB, have been shown to exist by many studies over the last few decades (Norris, Freeman \& Mighell 1996, hereafter NFM96; Suntzeff \& Kraft 1996; Lee et al. 1999; Pancino et al. 2000; Rey et al. 2004; Sollima et al. 2005a). There also exist ranges in abundance for all the elements studied in the cluster (Norris \& Da Costa 1995; Smith et al. 1995, 2000). These studies have primarily concentrated on the RGB stars as they are brighter than main sequence (MS) or subgiant ones.

There is evidence of an age range in $\omega$ Cen from the abundance and photometric studies. Specifically, the observed abundance patterns of different elements show the signatures of a variety of enrichment processes (Lloyd Evans 1977; Norris \& Da Costa 1995; Smith et al. 1995; Pancino et al. 2002). Contributing sources include Type II supernovae which result from high mass stars, asymptotic giant branch stars (AGB) that lose their material as stellar winds, and Type Ia supernovae - formed from older stars via mass transfer onto a white dwarf. Enrichment of s-process elements is seen in the RGB stars indicating contributions by low mass ( 1.5-3 solar masses) AGB stars (Lloyd Evans 1977; Norris \& Da Costa 1995; Smith et al. 1995, 2000). These AGB stars have lifetimes of order 1-3 Gyrs, indicating that there was an extended period over which enrichment and formation of the stars occurred in $\omega$ Cen. Results from Pancino et al. (2002) show a decrease in [Ca/Fe] at higher metallicities in the cluster. This indicates there are contributions from Type Ia supernovae in the enrichment processes, and again that it took place over an extended period.

Using Strömgren photometry, Hughes \& Wallerstein (2000) and Hilker \& Richtler (2000) examined the metallicity distribution and determined ages for samples of stars near the turnoff region in $\omega$ Cen. Both studies concluded that the more metal-rich stars in the cluster were younger than the metal-poor ones, with an age range of several gigayears.

Recently, high precision photometry of the cluster has shown perhaps as many as five discrete RGBs (Rey et al. 2004; Sollima et al. 2005a). An age range of $\sim 4$ Gyrs was determined using population modeling of the horizontal branch (HB) by Rey et al. (2004). Sollima et al. (2005a) obtained an upper limit to the range of 6 Gyrs using the RGB bumps which correspond to the different populations. The position of the bump in the RGB luminosity function on the CMD is a function of metallicity and age (and helium).

Ferraro et al. (2004) have shown from their high resolution images that a distinct metalrich subgiant branch (SGB) exists (designated SGB-a). Their conclusion from isochrone fitting the main metal-poor population and the SGB-a was that they are of the same age, indicating no age range in the cluster at all. The distinct SGB-a is also present in the 
photometric data of Bedin et al. (2004). Bedin et al. (2004) also find two distinct main sequences but surprisingly the red sequence contains $\sim 75 \%$ of the stars. On the RGB the majority of the stars lie along the blue side of that branch and it has been shown that the ratio of metal-poor to metal-rich objects is 80:20 (NFM96). This indicates the bluer sequence is the more metal-rich population, as has been confirmed spectroscopically by Piotto et al. (2004). The separation between the two sequences can be explained by the populations having significantly different helium abundances $(\Delta Y \approx 0.12$ ) (Bedin et al. 2004; Norris 2004; Lee et al. 2005). The source of this unusually high helium abundance in the metal-rich population is not clear.

Hilker et al. (2004) measured abundances of $\sim 400$ subgiant and turnoff stars using medium resolution spectra. Their abundance distribution resembles that from the RGB. Ages were derived for each star using its metallicity and position on the color-magnitude diagram (CMD) giving an age-metallicity diagram. They concluded from this diagram that a range of about 3 Gyrs exists in the cluster. Recently Sollima et al. (2005b) obtained VLT data of $\sim 250$ stars on the subgiant branches in $\omega$ Cen. They found an age range of no more than 2 Gyrs fitting isochrones to the populations in the cluster.

It has been suggested by Freyhammer et al. (2005) that the stars in the most metalrich population are actually located in a clump beyond the bulk of the cluster. Photometry for this metal-rich population was fitted with isochrones with metallicities in the range $1.1 \leq[\mathrm{Fe} / \mathrm{H}] \leq-0.8$ and using a larger distance modulus and reddening than is conventionally used for $\omega$ Cen. The direct spectroscopic abundance measurements of Sollima et al. (2005b) for stars along the metal-rich subgiant branch found their average metallicity to be $[\mathrm{Fe} / \mathrm{H}] \approx-$ 0.6, casting doubt on the result of Freyhammer et al. (2005).

In order to more accurately define the age range in the cluster, we have observed a sample of MS and turnoff (MSTO) stars. This sample will enable several new insights into the cluster since we have studied the MS stars spectroscopically. We have used these data to compare the abundance patterns and abnormalities found at the MSTO with those found for the RGB stars. The metallicity distribution on the MS has also been compared to that found on the RGB. A comparison of our MSTO sample with stars on the RGB can also show whether the enrichment of s-process and CNO elements is due to surface contamination, which would be obliterated by the growing convective envelope as the stars move on to the giant branch, or if the enrichment is uniform throughout the stars.

The major goal of the present work, however, is to look at the turnoff region of the CMD along with the metallicities for the members and determine a more accurate age range for the cluster. Most previous work at the turnoff region has utilized only photometric data. A spectroscopic approach coupled with photometry may prove to give a more accurate age 
range for the cluster and show any age-metallicity relation that exists. In $\S 2$ we describe the observations and reduction techniques. $\S 3$ outlines the derivation of metallicities for the sample. The discussion on the calculation of ages is described in $\S 4$, and $\S 5$ summarizes the results and comparisons with previous investigations. Preliminary accounts of these results have appeared in Stanford et al (2004).

\section{Observations and Reduction}

Photometry for the cluster was obtained with the 1m telescope/Tektronix CCD combination at Siding Spring Observatory, in the $V$ and $B$ bands. Ten fields with centers approximately 20 arcminutes from the cluster center were observed. Each field was $20 \times 20$ arcminutes in area. Exposure times were 500 seconds for the $V$ band and 900 seconds for the $B$ band. Typical seeing ranged between 1.8-2.2 arcseconds. The photometry was carried out using aperture photometry and the final sample only contained uncrowded stars (i.e. there were no neighbors within 5 arcseconds). The fields overlapped slightly in order to calibrate the frames in position and photometry. The photometry calibration used magnitudes from lists in the $B$ and $V$ bands from Cannon \& Stobie (1973a), Cannon (1981), and Cannon \& Stewart (1981) for objects that were in common. The photometric zero point uncertainties are of order 0.02 in both bands, and all errors come from photon statistics.

Preliminary positions were based on an early version of the US Naval Observatory catalog. These were then used to match stars from the SuperCosmos scan of a UK Schmidt

plate centered on $\omega$ Cen and positions of all stars were found. The final catalog positions from the SuperCosmos scan have an accuracy of $\sim 0.2$ arcseconds.

From these data a CMD, shown in Figure 1, was constructed for objects within an annulus 15-25 arcminutes from the cluster center. As there is no membership information, Figure 1 contains objects that belong to both $\omega$ Cen and the field. The Yonsei-Yale $\left(\mathrm{Y}^{2}\right)$ isochrones (Yi et al. 2001) were plotted along with the data and have metallicities $[\mathrm{Fe} / \mathrm{H}]=-$ $1.7,-1.2$, and -0.6 ; all have an age of 13.5 Gyrs. Abundance studies have determined that the different stellar populations in $\omega$ Cen show $\alpha$-enhancement (Norris \& Da Costa 1995; Smith et al. 1995, 2000; Pancino et al. 2002; Origlia et al. 2003). For metallicities $[\mathrm{Fe} / \mathrm{H}]=-$ 1.7 and $-1.2,[\alpha / \mathrm{Fe}]$ was taken to be 0.3 , and for $[\mathrm{Fe} / \mathrm{H}]=-0.6,[\alpha / \mathrm{Fe}]=0.18$ was used. In Figure 1 a reddening $E(B-V)=0.11$ (Lub 2001) and distance modulus $(\mathrm{m}-\mathrm{M})_{V}=14.10$ were assumed. This value of the distance modulus comes from Rey et al. (2004) and when fitting isochrones best reproduces the data. Mean photometric errors as a function of $V$ magnitude from the aperture photometry are shown in Table 1. 
In 1998 an area on the CMD was defined on the upper MS $(18.05 \leq V \leq 18.55 ; 0.30 \leq B-$ $V \leq 0.72$ ) with a view to determining the metallicity range. Stars in this region were observed using the Two degree Field Multiobject spectrograph (2dF) on the Anglo-Australian Telescope (Lewis et al. 2002). This spectrograph has the capability of simultaneously observing up to 400 objects using a fibre fed system. In 2002 a second region was defined at the turnoff $(17.25 \leq V \leq 18.5 ; 0.6 \leq B-V \leq 1.1)$ to look at the most metal-rich stars in the cluster and to determine the age range in the cluster, since the age degeneracy of the isochrones for a given metallicity can best be broken at the turnoff region. The first sample was observed in May 1998 and April 1999 (hereafter 98/99 sample), and the second in March 2002 (hereafter 2002 sample). Figure 1 shows the two boxes from which candidates were selected.

Although $2 \mathrm{dF}$ is able to observe 400 objects at once, for our sample the maximum number of objects we were able to observe per configuration was about 280 . This was due to the compact nature of our fields relative to the large field-of-view of the instrument, as well as the limit on fibre-to-fibre spacing. 1200 line/mm gratings were employed and the spectra obtained covered the useful wavelength range $\lambda \lambda 3800-4600 \AA$, with a scale of $1.1 \AA /$ pix. They have a resolution of $\sim 2.4 \AA$ FWHM.

As the number of probable members in the 1998 sample was high these objects were observed in one configuration for several hours. For the 1999 observations, the small number of non-members found in the previous run were removed and other candidates added to the configuration along with the confirmed members. This single configuration was again observed for several hours.

A slightly more complicated approach was taken for the 2002 observations. This sample extended to much redder colors to ensure than any high metallicity cluster members were included, but consequently had higher field star contamination. In order to completely observe our 2002 sample of 900 stars, a number of fibre configurations were needed and each of these contained successively fewer new stars due to crowding. The first step in the observing process was to determine which stars were members of the cluster. $\omega$ Cen has a large radial velocity of $\sim 232 \pm 0.7 \mathrm{kms}^{-1}$ (Dinescu et al. 1999a) while the field stars have velocities $\sim 0 \pm 50 \mathrm{kms}^{-1}$. This information was used to determine membership of the cluster. Each configuration was observed until a signal-to-noise ratio per pixel $(\mathrm{S} / \mathrm{N})$ of about 10 was reached. The exposure time depended on the weather and seeing, although the average was 1-2 hrs per field. The observations were carried out in half hour exposures to facilitate removal of cosmic rays. These data were then reduced and wavelength calibrated using the standard $2 \mathrm{dF}$ reduction software available at the telescope. The reduced fields were coadded and the individual spectra were then extracted using the Figaro routine EXTRACT. Once reduced and extracted the spectra were cross correlated with a spectrum of a previously 
confirmed $\omega$ Cen member in the IRAF package RV using FXCOR to obtain velocities. These were plotted as a histogram and membership was classified above a generous velocity cutoff limit.

Once we had observed all stars in our sample, the cluster members were re-observed with $2 \mathrm{dF}$ for $\sim 3-5$ hours in order to obtain higher $\mathrm{S} / \mathrm{N}$ spectra for more detailed analysis. The spectra were again cross-correlated, this time with a synthetic spectrum, to obtain velocities to confirm membership and obtain more accurate velocities. Analysis of twilight sky observations taken at the same time showed an offset in the velocities between the two CCD cameras of $\sim 11 \mathrm{kms}^{-1}$. A correction was applied to the spectra to account for the offset, and the individual spectra then coadded. The final heliocentric velocity histogram is shown in Figure 2. The narrow peak at $235 \mathrm{kms}^{-1}$ comprises the $\omega$ Cen members while the broader peak at lower velocities contains the field stars. The standard deviation was determined by an iterative $\pm 3 \sigma$ cutoff process (where $\sigma=13 \mathrm{kms}^{-1}$ ). The velocity dispersion in the outer regions of the cluster is low $\left(\sim 9 \mathrm{kms}^{-1}\right)$ (Merritt, Meylan, \& Mayor 1997), and the standard deviation is driven by both the velocity error measurement and dispersion. The mean velocity errors are typically $8 \mathrm{kms}^{-1}$. The standard deviation of the field stars was $\sim 50 \mathrm{kms}^{-1}$.

The 98/99 and 2002 samples were observed to completeness levels of $37 \%$ and $94 \%$ respectively, where the completeness level is defined as the ratio of number of objects observed to the number that had the potential to be observed. To be classed as "observable" each star underwent a visual inspection on the CCD images to ensure there were no contaminating objects within five arcseconds.

These processes yielded a final sample of 442 members from $~ 850$ observed candidates near the turnoff in the CMD of $\omega$ Cen. A CMD showing the members is shown in Figure 3 (large dots). The small dots represent the photometry as in Figure 1. The isochrones are the same as those in Figure 1. This figure shows that there are a number of very red, presumably metal-rich, stars in $\omega$ Cen at the turnoff region. Objects that were classified as radial velocity non-members of the cluster in the 2002 sample are shown in Figure 4. This diagram shows that the candidates were positioned fairly uniformly over the 2002 region on the CMD. It also makes clear that while we found no members in the lower right corner of the box in Figure 3, candidates were observed there. Tables 2 and 3 briefly lists the members and non-members, respectively. Both Tables are shown in their entirety in the electronic version of The Astrophysical Journal. Table 2 details the identification (ID), right ascension (RA) and declination (DEC) of each cluster member, along with the $\mathrm{V}$ magnitude and color $(B-V)$. Column 6 lists the heliocentric velocity. Columns 7 and 8 list the determined metallicity $([\mathrm{Fe} / \mathrm{H}])$ and the error associated with it $\left(\sigma_{[\mathrm{Fe} / \mathrm{H}]}\right)$. Columns 9 and 10 give the 
age assigned to each star and its error. Finally, column 11 informs the reader on which run the star was observed, where 1 is for the 98/99 sample and 2 for 2002. Table 3 gives the identification (ID), right ascension (RA) and declination (DEC) of the non-members, and photometry information. Column 6 lists the heliocentric velocity, and the final column states on which observing run the star was observed.

Example spectra of a metal-poor subgiant, whose metallicity is representative of the majority of the cluster population, and one of the more unusual metal-rich member are shown in Figure 5. Noticeable differences between the two spectra are the increased G band between $4250-4310 \AA, C N$ at $3883 \AA$ and $4215 \AA$ and numerous metal lines in the more metalrich star. A paper on the analysis of the abundances of carbon, nitrogen, strontium and barium for all stars, including the more peculiar objects, is in preparation.

Spectra with $2 \mathrm{dF}$ were also obtained for main sequences stars in four other globular clusters, NGC 6397, M55, NGC 6752 and 47 Tuc. These clusters were observed in a similar manner to $\omega$ Cen. The data obtained for the clusters were used here to test the reliability of the metallicity calibration. Observing dates and parameters used are given in Table 4. Abundance standards were also observed for calibrations during each $2 \mathrm{dF}$ cluster run.

\section{Metallicities}

\subsection{Abundance Calibration}

Metallicities were calculated using a combination of two methods following Beers et al. (1999). The first uses the Ca II K line strength (in the form of a pseudo-equivalent width) and $(B-V)_{0}$. In order to take into account the large range in $\mathrm{K}$ line strength, three different on-feature band widths were used. These were 6,12 and $18 \AA$ and formed indices designated K6, K12 and K18 respectively. The line bands and sidebands for these indices are defined as in Beers et al. (1999). The final index, $K^{\prime}$, is given by:

$$
\mathrm{K}^{\prime}= \begin{cases}\mathrm{K} 6 & \mathrm{~K} 6 \leq 2.0 \AA \\ \mathrm{K} 12 & \mathrm{~K} 6>2.0 \AA, \mathrm{K} 12 \leq 5.0 \AA \\ \mathrm{K} 18 & \mathrm{~K} 12>5.0 \AA\end{cases}
$$

This metallicity determination becomes more uncertain at higher metallicities due to the saturation of the Ca II $\mathrm{K}$ line. Another uncertainty arises from $(B-V)_{0}$, as this color index may be affected by anomalous $\mathrm{CN}$ absorption not found in the calibrating objects. At fixed $\mathrm{K}^{\prime}$ and for a $(B-V)_{0}$ typical of the more metal-rich stars that show strong $\mathrm{CN}$ 
absorption, a change of $(B-V)_{0}$ of \pm 0.02 mag results in an abundance change of \pm 0.08 dex.

The second method used a technique known as the Auto-Correlation Function (ACF) method which utilizes the strength of the metal lines in the spectrum. The wavelength range used in this technique is $\lambda \lambda 4000-4285 \AA$ excising the CN band and hydrogen lines in the process (from 4166-4216 and $20 \AA$ centered on $\mathrm{H}_{\delta}$ ). The main drawback of this calibration is that the spectra need to have a sufficient signal-to-noise ratio (at least $\mathrm{S} / \mathrm{N} \approx 20$ ) so that the method is not seriously affected by noise.

To quantify the usefulness of the two indices, tests were performed to determine cutoff limits in $\mathrm{S} / \mathrm{N}$ and in $(B-V)_{0}$ for which only $\mathrm{K}^{\prime}$, only $\mathrm{ACF}$, or the combination of both would be used. The cutoff limits were determined from spectra of 15 high proper motion stars chosen from the lists of Giclas (see e.g. Carney et al. 1996) to cover the range in metallicities found in $\omega$ Cen. Spectra of these objects, which are all dwarfs, were obtained with ANU's 2.3m telescope at Siding Spring Observatory and have a resolution of $1.2 \AA$ FWHM and $\mathrm{S} / \mathrm{N} \sim 100$. The spectra were convolved to have the same resolution as our $2 \mathrm{dF}$ sample and five different levels of noise were introduced to the spectra to cover the $\mathrm{S} / \mathrm{N}$ range in our sample. The convolved and noise-added spectra were then analyzed with our metallicity determination technique, and the resulting abundances compared, as a function of color and $\mathrm{S} / \mathrm{N}$, with the abundances that result from applying our technique to the original higher resolution, high $\mathrm{S} / \mathrm{N}$ spectra. As expected, objects with higher metallicities showed larger errors when using the $\mathrm{K}^{\prime}$ index, while the metal-poor stars had larger scatter when ACF was employed.

This procedure also found a systematic offset in the ACF abundance in the sense that the abundances derived from the convolved noise-added spectra were higher than those for the original spectra. The offsets were a function of metallicity, color and $\mathrm{S} / \mathrm{N}$, with bluer objects having larger offsets than redder ones for a given S/N. Given the similarity between the convolved noise-added spectra of the Giclas dwarfs and those of our $\omega$ Cen stars, we applied these corrections, again as a function of metallicity, color and $\mathrm{S} / \mathrm{N}$, to the initial $\omega$ Cen star ACF metallicity determinations to account for this systematic effect. The corrections ranged in size from -0.1 to -0.3 dex.

We also analyzed $2 \mathrm{dF}$ observations of a number of field dwarfs on known metallicities chosen from the lists of Carney et al. (1996) and Beers et al. (1999). Approximately half of these spectra were obtained during our cluster observing runs while the remainder were obtained from other runs using the same instrumental setup. Our $\mathrm{K}^{\prime}$ and ACF abundances derived from these spectra generally agreed well with the literature values: the mean differences were $\sim 0.1$ dex for $[\mathrm{Fe} / \mathrm{H}]<-1.0$, but somewhat higher $(\sim 0.2-0.3 \mathrm{dex})$ for the more metal-rich objects. 
The existence of $2 \mathrm{dF}$ spectra for substantial samples of main sequence stars in four globular clusters (NGC 6397, M55, NGC 6752 and 47 Tuc) allows a further investigation of our abundance determinations. These spectra have similar $\mathrm{S} / \mathrm{N}$ to our $\omega$ Cen sample. We applied our technique to the cluster main sequence star spectra and the resulting abundance histograms for $[\mathrm{Fe} / \mathrm{H}]_{K^{\prime}}$ and $[\mathrm{Fe} / \mathrm{H}]_{A}$ are shown in Figures 6 and 7 respectively. In the case of $[\mathrm{Fe} / \mathrm{H}]_{K^{\prime}}$, we find that the mean abundances for the clusters from our technique are systematically low by 0.2 to 0.3 dex compared to the accepted metallicities (Harris 1996). We note also that the distribution for 47 Tuc is considerably broader than for the other three clusters. This results from the saturation of the Ca II K line at higher in metallicities, which, in turn, causes a larger uncertainty in the final $[\mathrm{Fe} / \mathrm{H}]_{K^{\prime}}$. Further, we verified that the possible bimodality of the 47 Tuc abundance distribution in Figure 6 is not the results of the known bimodality in $\mathrm{CN}$ strengths on the cluster main sequence.

For the ACF metallicity, despite use of the offsets defined from the analysis of the Giclas star spectra, the values for NGC 6397 and 47 Tuc are higher than the accepted values by $\sim 0.2$ dex $\left(\right.$ no $[\mathrm{Fe} / \mathrm{H}]_{A}$ values were derived for the M55 and NGC 6752 stars as the spectra generally do not possess sufficient counts to apply the technique). We note also that the width of the NGC $6397[\mathrm{Fe} / \mathrm{H}]_{A}$ distribution is quite broad. This is a consequence of the relatively low sensitivity of the ACF method at low abundance.

It is not clear why the clusters give systematic offsets in the $[\mathrm{Fe} / \mathrm{H}]_{K^{\prime}}$ and $[\mathrm{Fe} / \mathrm{H}]_{A}$ abundances while the standard stars do not, although it may be that we simply do not have enough standard objects to thoroughly test for any offsets. Our preference is to use the cluster data rather than that of the standard stars given the much larger sample sizes in each cluster at a given $[\mathrm{Fe} / \mathrm{H}]$ (hundreds versus a few). It was also considered better to use objects that were similar in magnitude and color and were observed and reduced in the same manner as the $\omega$ Cen stars.

Comparisons between the mean $\mathrm{K}^{\prime}$ metallicities for each cluster and the accepted $[\mathrm{Fe} / \mathrm{H}]$ values are shown in Figure 8, along with a similar comparison for the ACF metallicities. The median of the distributions for 47 Tuc and for NGC $6397\left([\mathrm{Fe} / \mathrm{H}]_{A}\right)$ were used here. In these plots the dotted lines are 1:1 relations. In the upper panel, the solid line is the least-squares fit to the data, while the lower panel's solid line is an offset of 0.2 dex to the $1: 1$ line. Corrections to the $\mathrm{K}^{\prime}$ metallicities were constructed based on a linear fit from the calibrating clusters and were applied to the $\omega$ Cen data. The ACF correction used was an 0.2 dex offset from the 1:1 line. Fitting a straight line to the ACF data may not give an accurate correction as there are only two data points and to err on the side of caution, we instead used an offset. 


$$
\begin{aligned}
{[\mathrm{Fe} / \mathrm{H}]_{\mathrm{K}^{\prime} \mathrm{c}} } & =\frac{[\mathrm{Fe} / \mathrm{H}]_{\mathrm{K}^{\prime}}+0.17}{1.06} \\
{[\mathrm{Fe} / \mathrm{H}]_{\mathrm{Ac}} } & =[\mathrm{Fe} / \mathrm{H}]_{\mathrm{A}}-0.2
\end{aligned}
$$

The $\mathrm{K}^{\prime}$ calibration is more reliable for metal-poor stars due to the saturation of the $\mathrm{Ca}$ II $\mathrm{K}$ line at higher metallicities. The ACF method, on the other hand, is more reliable for the metal-rich objects due to the loss of sensitivity at lower abundances. Therefore limits were put in place at metal-poor and metal-rich ends of our metallicity range to use only the method that suited best. The final metallicity was given by:

$$
[\mathrm{Fe} / \mathrm{H}]= \begin{cases}{[\mathrm{Fe} / \mathrm{H}]_{\mathrm{K}^{\prime} \mathrm{c}}} & {[\mathrm{Fe} / \mathrm{H}]_{\mathrm{K}^{\prime} \mathrm{c}} \text { or }[\mathrm{Fe} / \mathrm{H}]_{\mathrm{Ac}} \leq-2.0} \\ {[\mathrm{Fe} / \mathrm{H}]_{\mathrm{Ac}}} & {[\mathrm{Fe} / \mathrm{H}]_{\mathrm{K}^{\prime} \mathrm{c}} \text { or }[\mathrm{Fe} / \mathrm{H}]_{\mathrm{Ac}} \geq-0.8} \\ \langle[\mathrm{Fe} / \mathrm{H}]\rangle & \text { otherwise }\end{cases}
$$

The weighted mean of the two metallicities was calculated using:

$$
\langle[\mathrm{Fe} / \mathrm{H}]\rangle=\frac{\frac{[\mathrm{Fe} / \mathrm{H}]_{\mathrm{K}^{\prime} \mathrm{c}}}{\sigma_{\mathrm{K}^{\prime} \mathrm{c}}}+\frac{[\mathrm{Fe} / \mathrm{H}]_{\mathrm{Ac}}}{\sigma_{\mathrm{Ac}}^{2}}}{\frac{1}{\sigma_{\mathrm{K}^{\prime} \mathrm{c}}^{2}}+\frac{1}{\sigma_{\mathrm{Ac}}^{2}}}
$$

The error estimates associated with the initial $\mathrm{K}^{\prime}$ and $\mathrm{ACF}$ determinations were derived from two sources. The first is from the Beers formulation itself, where an error estimate is assigned for the $\mathrm{K}^{\prime}$ and ACF metallicities individually as described in Beers et al. (1999). The second source comes from the fact that the $\mathrm{S} / \mathrm{N}$ of our data is lower than the average $\mathrm{S} / \mathrm{N}$ of the spectra used in the Beers calibration. This takes account of the fact that the ACF metallicities are impacted more by the noise level in the spectra, which is particularly the case for the metal-poor stars. Using the procedure to correct the metallicities described earlier, errors were assigned to the metallicities based on the stars color, metallicity and S/N $(\sigma \leq 0.2$ dex). These two sources were added quadratically to give the error estimate.

An independent estimate of the errors used the data from the calibrating clusters. Two of the calibrating clusters have metallicities which are approximately equal to our outer boundaries at $[\mathrm{Fe} / \mathrm{H}]=-2.0$ and -0.8 . The standard deviation was calculated for the metallicity distribution for these clusters and adopted as the error $(\sigma)$ for the $\omega$ Cen stars at the metallicity of the cluster. For NGC 6397 and 47 Tuc, $\sigma_{K^{\prime}}=0.14$ and 0.29 , and $\sigma_{A C F}=0.30$ and 0.27 , respectively. Linear interpolation was used between the two metallicities in order 
to assign errors at all metallicities between our boundary limits. This technique was performed for both ACF and $\mathrm{K}^{\prime}$ abundances. At lower metallicities the standard deviation for $\mathrm{K}^{\prime}$ was lower than that for ACF. Conversely the ACF standard deviation was lower at higher metallicities than $\mathrm{K}^{\prime}$.

For the $\mathrm{K}^{\prime}$ abundance error, the first estimate was higher than the second by $\leq 0.1$ dex. The opposite was true, however, for the ACF metallicity error by the same amount. For both $\mathrm{K}_{c}^{\prime}$ and $\mathrm{ACF}_{c}$ the final adopted error was taken as the average of the two separate estimates, while the overall error associated with a given metallicity was taken as the quadratic sum:

$$
\frac{1}{\sigma_{\mathrm{F}}^{2}}=\left(\frac{1}{\sigma_{\mathrm{K}^{\prime} \mathrm{c}}^{2}}+\frac{1}{\sigma_{\mathrm{Ac}}^{2}}\right)
$$

\subsection{Metallicity Distribution}

Figure 9 shows the resulting metallicity distributions obtained for the 98/99 (panel a) and 2002 samples (panel b). The distributions are compared with that found for the RGB of the cluster, taken from NFM96. To convert their $[\mathrm{Ca} / \mathrm{H}]$ distribution to $[\mathrm{Fe} / \mathrm{H}],[\mathrm{Ca} / \mathrm{Fe}]$ was assumed to be 0.3 for $[\mathrm{Fe} / \mathrm{H}] \leq-1.0$ (Smith et al. 1995; Pancino et al. 2000), declining linearly from $[\mathrm{Ca} / \mathrm{Fe}]=0.3$ at $[\mathrm{Fe} / \mathrm{H}]=-1.0$ to 0.0 for $[\mathrm{Fe} / \mathrm{H}]=0$. The distributions have been normalized by area and the RGB distribution has been convolved with a wider gaussian kernal $(\sigma=0.14)$ than in NFM96 due to the larger errors associated with our metallicities. The metallicity errors for our sample are $\sim 0.15-0.2$ dex, compared with 0.05 dex for that of the NFM96. The generalized histograms for the 98/99 and 2002 samples utilize the individual $\sigma$ associated with each metallicity.

The 98/99 spectra were of hotter and fainter objects. The range of $\mathrm{S} / \mathrm{N}$ for the 2002 sample was $\sim 30-70$, while for the $98 / 99$ spectra it was $\sim 20-40$. The lower $\mathrm{S} / \mathrm{N}$ for the $98 / 99$ sample and the fact that the majority had metallicities calculated using the $\mathrm{K}^{\prime}$ calibration instead of the combination of the ACF and $\mathrm{K}^{\prime}$ calibrations made their metallicity determinations slightly more uncertain. Since it is the 2002 sample, in particular the brighter stars at the turnoff, that gives us the most information about the age range in the cluster the larger errors in abundance on the main-sequence stars are not a great concern for the age determinations.

The data from our two sub-samples were not combined due to the 2002 set being incomplete at the metal-poor end and biased towards the metal-rich populations. The 98/99 sample is unbiased with respect to the distribution of members in the CMD (except for a 
small number of stars with $B-V>0.72$ ), while the 2002 one is biased against the metalpoor sample. This can be seen in the offset between the 2002 sample and the NFM96 data, as no corrections have been made for selection effects. There have also been no evolutionary corrections made to the distributions, though these are likely to be minor.

The distributions have a steep rise at $[\mathrm{Fe} / \mathrm{H}]=-1.7$, with tails to higher metallicities. We find $25 \%(44 / 174)$ and $15 \%(39 / 254)$ of stars with metallicities $[\mathrm{Fe} / \mathrm{H}]<-1.7$ for the $98 / 99$ and 2002 samples, respectively. Stars with $[\mathrm{Fe} / \mathrm{H}]>-1.0$ account for $4 \%(11 / 254)$ for the 2002 sample and $5 \%$ (8/174) for the 98/99 sample. In the NFM data there are $25 \%$ of stars with $[\mathrm{Fe} / \mathrm{H}]<-1.7$ and $6 \%$ of stars with $[\mathrm{Fe} / \mathrm{H}]>-1.0$. The reader should note that the NFM sample covers the whole region of the cluster, while our sample covers the outer region between 15 and 25 arcminutes. We conclude that the metallicity range found for the turnoff region is qualitatively similar to that found for the giant branch.

A Kolmogorov-Smirnov two-sample test was performed on the 98/99 and NFM96 distributions. The null hypothesis was that the two samples came from the same distribution. This test was also repeated using the data sets from Suntzeff \& Kraft (1996) in place of that of NFM96. Their data for $\omega$ Cen comprise two groups, one of subgiant branch objects and the other of red giant branch stars. These three sets of data were tested against our 98/99 data separately. For the 98/99 data, we found that the null hypothesis could not be rejected. Not surprisingly given the biased selection, the 2002 data set showed a different result, and the null hypothesis was rejected for each of the three tests.

To check the accuracy of the metallicities the members falling in the 2002 turnoff box were separated into three groups based on their photometry shown in the lower panels of Figure 10. This figure illustrates the differences in metallicity as a function of position on the CMD. The solid lines indicate where the regions of interest lie. These lines are based on an isochrone where the first (from the left) has parameters of $[\mathrm{Fe} / \mathrm{H}]=-1.2$, age $=13.5$ Gyrs, and offset in $V$ by $-0.14 \mathrm{mag}$, and in $(B-V)$ by $-0.062 \mathrm{mag}$. The second solid line is the same isochrone but offset by $V=0.12 \mathrm{mag}$ and $(B-V)=0.018 \mathrm{mag}$.

Corresponding metallicity histograms for each group were plotted shown in the upper panels. The first group has a mean $[\mathrm{Fe} / \mathrm{H}]=-1.61 \pm 0.13$, the second $[\mathrm{Fe} / \mathrm{H}]=-1.48 \pm 0.17$. The third group has a mean $[\mathrm{Fe} / \mathrm{H}]=-1.28$, but note the small number of objects in this group. The errors in abundance for the third group are large $(0.3 \mathrm{dex})$, evident by the large width of the histogram. 


\section{Ages}

When determining the ages using theoretical isochrones, it is best to use the turnoff region since this is where the isochrones are more sensitive to age variations. For the present investigation only the members at the turnoff with $V \leq 18$ were used, and these stars came from the 2002 sample.

Two methods were used to calculate the age range of the cluster. The first involved assigning individual ages to each star based on its position on the CMD and metallicity using theoretical isochrones. The second method involved the construction of synthetic CMDs from a specified metallicity distribution, age range and theoretical isochrones, and comparison between synthetic and observed CMDs.

The isochrones used were the Yonsei-Yale $\left(\mathrm{Y}^{2}\right)$ isochrones (Yi et al. 2001; Kim et al. 2002). These isochrones permit interpolation between age, metallicity and alpha elemental abundance to generate the required isochrone. A grid of isochrones was used which span the metallicity range $-2.6<[\mathrm{Fe} / \mathrm{H}]<0.3$ in 0.05 dex increments. For each metallicity there were 34 isochrones for ages 2-19 Gyrs in 0.5 Gyr steps. Alpha enhancement was taken to be constant $([\alpha / \mathrm{Fe}]=0.3)$ for $[\mathrm{Fe} / \mathrm{H}] \leq-1.0$, and declining linearly for higher $[\mathrm{Fe} / \mathrm{H}]$ until it reached the solar value at $[\mathrm{Fe} / \mathrm{H}]=0$.

The RGB metallicity distribution from NFM96 was used as the input into all simulations when requiring synthetic CMDs in the following sections. This distribution was shown in the previous section to be similar to the one found on the main sequence. As the RGB distribution is for $[\mathrm{Ca} / \mathrm{H}]$ rather than $[\mathrm{Fe} / \mathrm{H}]$, it was scaled using constant $[\mathrm{Ca} / \mathrm{Fe}]=0.3$ for $[\mathrm{Fe} / \mathrm{H}]<-1.0$ and linearly decreasing $[\mathrm{Ca} / \mathrm{Fe}]$ to 0.0 at $[\mathrm{Fe} / \mathrm{H}]=0.0$ for $[\mathrm{Fe} / \mathrm{H}]$ greater than -1.0 .

\subsection{Method 1: Assigning Individual Ages to Stars}

To assign an age to each star, its metallicity was used to select the nearest isochrone in our grid. The isochrones with this metallicity but with differing ages were then compared to the star's $(B-V)_{0}$ and $\mathrm{M}_{V}$ on the CMD to find the one closest. Usually a star's position did not fall directly on one isochrone and linear interpolation in color was performed between the two closest ones to determine its age.

An error associated with the age was obtained using the errors in $B-V$ and metallicity. The errors associated with the individual $\mathrm{V}$ magnitude contributes a very small amount to the final error and was therefore ignored here. The age calculation was repeated for 
a positive and negative change in color using the values given in Table 1 . Similarly the metallicity was modified using $\pm 1 \sigma$ errors to obtain the corresponding error in age. The range in age determined by the metallicity errors and that determined by the photometry errors were quadratically summed and halved to give the final estimate of the age error.

Total errors in the age calculation were up to \pm 4 Gyrs for stars below the turnoff where the isochrones are close together, and up to \pm 2 Gyrs for objects above the turnoff. There were some stars that did not fit any of the isochrones in the grid for their metallicity. These were given the maximum (or minimum) age in the range i.e. 19 (or 2) Gyrs and represented $\sim 7 \%$ of the sample. An age of 19 Gyrs for a object in a globular cluster is not believable, nor is one of 2 Gyrs. This discrepancy is probably due to errors in the photometry or calculated metallicity.

Figure 11 shows the age-metallicity diagram (AMD) resulting from this method. This plot shows only those stars that lie at the turnoff region of the CMD with $V<18.0$, where the metallicity-age degeneracy is best broken. It shows that there is an age-metallicity relation in the cluster with the more metal-rich stars being younger. A line of best fit to the data was drawn, by eye, excluding stars at the upper age limit object (at 19 Gyrs) and is shown as the solid line. This fit gives an age range of $\sim 5$ Gyrs between $[\mathrm{Fe} / \mathrm{H}]=-1.7$ and -0.6 . As discussed below, however, this relation is influenced by correlated errors.

To test the age range found above we performed Monte-Carlo simulations of a population which had the metallicity distribution taken from NFM96. The synthetic population of stars occupied the same position on the CMD as the $\omega$ Cen turnoff stars in our sample, and had the same sample size. Four different age ranges were considered - 0, 2, 4 and 6 Gyrs between metallicities $[\mathrm{Fe} / \mathrm{H}]=-1.7$ and -0.6 , with a linear interpolation in age between these metallicities. The oldest population in each case was assigned an age of 13.5 Gyrs and $[\mathrm{Fe} / \mathrm{H}]=-1.7$. For example, the ages for the 2 Gyr age range simulation was calculated as follows:

$$
\text { Age }_{\star}= \begin{cases}13.5 & {[\mathrm{Fe} / \mathrm{H}]_{\star} \leq-1.7} \\ -1.82[\mathrm{Fe} / \mathrm{H}]_{\star}+10.41 & -1.7<[\mathrm{Fe} / \mathrm{H}]_{\star}<-0.6 \\ 11.5 & {[\mathrm{Fe} / \mathrm{H}]_{\star} \geq-0.6}\end{cases}
$$

Photometric errors were included that were representative of the observed sample (see Table 1). We also included an error on the abundance determination $(\sigma=0.15$ dex) when simulating the populations. We then determined ages for all stars in each of the populations in the same manner as was done for the observed data. The goal was to test how well we could recover the input parameters given the errors on photometry and metallicity. 
The resulting AMDs are shown in Figure 12. In these plots, each point represents a simulated star, and was assigned an age depending on the input parameters. Simulation 1 has no age range, simulations 2, 3 and 4 have age ranges of 2, 4 and 6 Gyrs, respectively, between metallicities $[\mathrm{Fe} / \mathrm{H}]=-1.7$ and -0.6 . Any stars that have abundances beyond those values have the maximum or minimum ages assigned to them. The dot-dash line in each plot indicates the input age-metallicity relation before any photometric or metallicity errors were included. The solid line is the least squares fit to the data, which takes errors in both coordinates into consideration.

The first thing to note in Figure 12 is that errors induce an age-metallicity relation. Panel a, which has an input age range of 0 Gyrs shows an apparent age range of 3.9 Gyrs. As the input age range becomes larger, the calculated age range comes more into line with it. The simulations with 4 and 6 Gyr age spreads do not accurately reproduce the observed AMD for $\omega$ Cen which can be seen by comparing Figure 12 with the observations in Figure 13 (the latter is similar to Figure 11 but without the error bars). For these simulations, the correlation between age and metallicity is too tight when compared with that of the $\omega$ Cen plot. The two simulations with age ranges of 0 and 2 Gyrs show a scatter that is similar to that of $\omega$ Cen, and have similar slopes. This shows that while the observational data indicate an age range of $\sim 5$ Gyrs, this figure drops considerably when the errors in metallicity and photometry are taken into account. From this we conclude that the age range in $\omega$ Cen lies between 0 and 2 Gyrs. To summarize: errors in metallicity and photometry have the potential to induce an unreal age-metallicity relation, or to make a small one appear larger.

These simulations also show the evolutionary effects for different metallicities and ages. Simulations with higher age ranges have more metal-rich objects than those with no or small age ranges. This is due to our choice of turnoff box and to metal-rich young stars spending a longer period within the box than the older metal-rich objects. As only a small number of metal-rich objects were found in our observed sample, the above result strengthens the case for a low age range within the cluster.

A further check of the age range found is to plot the data within metallicity ranges on the CMD and fit isochrones of appropriate metallicities and ages (all with $[\alpha / \mathrm{Fe}]=0.3$ ), shown in Figure 14. Four metallicity bins were chosen. The first had a mean metallicity of $[\mathrm{Fe} / \mathrm{H}]=-1.7$, and an appropriate isochrone with age 14 Gyrs was found to best fit the data. Isochrones with age of 12 and 16 Gyrs were also plotted as dotted lines to show the the approximate range in ages covered by the data. Group 2 had a mean metallicity of $[\mathrm{Fe} / \mathrm{H}]=-1.5$. There is a broader spread in metallicities in this group, accounting for the members that are not encompassed by the isochrones plotted. The third group had a mean metallicity of $[\mathrm{Fe} / \mathrm{H}]=-1.2$. Isochrones of this metallicity and ages $=13 \pm 2$ Gyrs were found 
to fit the data, although there are a few outliers in this group. The mean $[\mathrm{Fe} / \mathrm{H}]=-0.8$ of the fourth group was also used to plot isochrones with ages $=12 \pm 2$ Gyrs. This figure indicates that there is indeed a age range in the cluster.

\subsection{Helium abundance variations}

It has been suggested that large helium variations $(\Delta \mathrm{Y} \sim 0.12)$ play a key role in understanding the bimodality of the MS (Bedin et al. 2004; Norris 2004; Piotto et al. 2004). It is therefore important to see what effect helium variations have at the turnoff region of the CMD and the ages calculated for our sample. Unfortunately the $\mathrm{Y}^{2}$ isochrones (as well as most other sets of published isochrones) do not present results for sufficiently large ranges of helium for a given $\mathrm{Z}$. Ones that do cover the required range in helium and $\mathrm{Z}$ to date are the Revised Yale Isochrones (RYI) (Green, Demarque \& King 1987). While these models do not contain the most up-to-date physics, they are adequate to show the relative effects of helium variations.

To test the effect of helium we assumed our sample had two populations - the first had $[\mathrm{Fe} / \mathrm{H}] \leq-1.45$ while the second had $[\mathrm{Fe} / \mathrm{H}]>-1.45$. The standard value of $\mathrm{Y}(=0.23)$ was applied to the first population, and helium for the second population would then be 0.35 (for $\Delta Y=0.12$ ).

Although we wished to use $Y=0.23$ and $Y=0.35$ (for $\Delta Y=0.12$ ) for the first and second groups, respectively, the RYI are incomplete above $\mathrm{Y}=0.3$ and ages are not able to be calculated at the turnoff. Therefore values of $Y=0.2$ and 0.3 were used to show the relative effect (using $\Delta Y=0.10$ ). To test the effect of helium on the ages, two sets of isochrones were used. Both covered a metallicity range of $-2.6<[\mathrm{Fe} / \mathrm{H}]<-0.5$ and an age range of $6-20$ Gyrs. All the isochrones in the first set had $\mathrm{Y}=0.2$. For isochrones in the second set that had $[\mathrm{Fe} / \mathrm{H}] \leq-1.45$, helium was taken to be 0.2 , and for those with $[\mathrm{Fe} / \mathrm{H}]>-1.45, \mathrm{Y}=0.3$. Ages were calculated using these isochrones for each of our members in the cluster in the same manner as described is $\S 4.1$.

The results are shown in Figure 15. The two upper panels show the AMDs for $\Delta Y=0.0$ and 0.1 respectively. The lower panel compares the ages determined with and without helium variations. These plots show that there is little variation at the turnoff region for a particular star when changing its assumed helium abundance. Although there is a significant difference between the positions on the CMD at the MS and RGB, the turnoff region difference is very small. We conclude that the possible enhancement of helium in the second population does not significantly affect the ages calculated at the turnoff region. There is one star in which 
the ages do differ by a significant amount (age $=15$ Gyrs for $\mathrm{Y}=0.2$ and age $=20$ Gyrs for $Y=0.3$ ). This is due to its position on the CMD. It is at the SGB as opposed to the turnoff region, and here the isochrones lie very close together and small variations in position of the isochrones for the two sets induce a large age variation.

\subsection{Method 2: Synthetic Color Magnitude Diagrams}

The second method to determine the age range in $\omega$ Cen utilized only the photometry information. No use was made of the spectroscopy data other than it supports the use of NFM96 as the abundance distribution. Synthetic color-magnitude diagrams were constructed using the $\mathrm{Y}^{2}$ isochrones and the metallicity distribution from NFM96. Monte Carlo simulations were performed to obtain simulations with linear age ranges between 0 and 8 Gyrs, in 0.5 Gyr increments. The age ranges were applied between the metallicities $[\mathrm{Fe} / \mathrm{H}]=-1.7$ and -0.6 . Any points that had metallicities outside this range were assigned the maximum or minimum value, with the maximum age being 13.5 Gyrs in each simulation. Errors in photometry were assigned to each point, in accordance with the data in Table 1. Each simulation had $N=80000$. These simulations did not include binary stars.

Four of the CMDs obtained from these simulations are shown in Figure 16. Each CMD shows the synthetic points, with the objects with $V \leq 18$ falling in the 2002 turnoff box as larger symbols. Figure 17 shows the $\omega$ Cen data and is for comparison purposes. Panel a is for a simulation with no age spread; b, c, and d are for age spreads of 2, 4 and 6 Gyrs, respectively, between metallicities $[\mathrm{Fe} / \mathrm{H}]=-1.7$ and -0.6 . In order to objectively test which simulation represented our observed sample best, a $\chi^{2}$ test was performed. This involved dividing the CMD where our objects lie into smaller boxes. A grid of $3 \times 5$ boxes (three in $V$ magnitude and five in $B-V$ ) was used in this case, and each box was given equal weighting. We established, through a series of tests using different numbers of boxes, that the $3 \times 5$ grid gave the best chance of finding the age range.

In order to accurately interpret the results of the $\chi^{2}$ fitting we first tested our simulations and statistical calculations. The first test involved using a sample from one of the simulations to represent our observed data. The objects were chosen randomly and had the same sample size as our observed sample $(n=222)$. This was repeated five times to check the consistency of the results. Four representative samples were chosen with ages spreads of $0,2,4$ and 6 Gyrs. These were tested against all the other, larger N, simulations in the same manner as the observed sample to see which simulation was the "best fit". The best result in these cases would of course be the input age range. For example, the 2 Gyr representative sample should be best reproduced by the 2 Gyr simulation. The results of these tests are shown 
in Figure 18. For the representative sample with no age range, panel a in Figure 18, the statistical test seems to find the correct result, although an age range of 2 Gyrs can not be definitively ruled out. The 2 Gyr representative sample (panel b) does not have a clear solution as to which simulation is best represented by it and the test shows that it could be anywhere between 0 and 4 Gyrs. The test does reproduce an age range of 4 Gyrs for

the 4 Gyr representative sample (panel c), but again there is uncertainty of \pm 2 Gyrs. The representative sample with a 6 Gyr age spread seems to be the most clearly defined result and accurately predicts an age range of 6 Gyrs for the sample (panel d). These tests indicate that the higher the age range the more likely it will be recovered by this statistical test. This effect was also seen in the simulations done in $\S 4.1$, and shown in Figure 12. Lower age ranges are harder to identify than larger ones.

The $\chi^{2}$ results for $\omega$ Cen are shown in Figure 19. Comparing these to the tests performed on the representative samples, we can see that the $\omega$ Cen results are similar to the 4 Gyr results. Both have an age range of 4 Gyrs as the lowest $\chi^{2}$ value, and both have small values for the 6 Gyr simulation as well. The $\chi^{2}$ result for the 2 Gyr age range is somewhat higher than in the 4 Gyr graph, and resembles the 6 Gyr representative sample results with a steep slope at low age ranges. The shape of the curve indicates that the age range is not 0 or 2 Gyrs, but could possibly be up to 6 Gyrs. We can rule out large age ranges (of the order 6 Gyrs) as the $\omega$ Cen data do not show a distinct result for it in these simulations, nor in $\S 4.1$.

\subsection{Simulations with no age-metallicity relation}

Simulations were also produced that had the $\omega$ Cen metallicity distribution and a range in ages but no age-metallicity relation, examples of which are shown in Figure 20. The ages are chosen randomly for each object, and as such had a flat distribution. This figure shows four simulations with age ranges of 0, 2, 4 and 6 Gyrs. The 2002 CMD limits are also shown, and the simulated objects falling in this area are highlighted. The solid line in the figures represents the blue edge fiducial of the $\omega$ Cen data (see Figure 17).

These simulations did not match the 2002 sample. In particular the old metal-rich stars and young metal-poor stars were found in the simulations but are not seen in the observational data. The young metal-poor stars in the simulations are seen in the bluer and brighter turnoff region which have no counterpart in the observed sample as shown in Figure 17. The old metal-rich stars are found in the bottom right corner of the 2002 box in the CMD and again there were none of these stars found in the 2002 sample. This last result cannot be explained by a lack of candidate members being observed in this area. The 
simulated stars were well distributed across this region, but no members of $\omega$ Cen were found in that area. Although some of these simulations might be considered as fitting the observed data, it is the presence of young metal-poor stars at and above the turnoff that excludes this scenario. This does indicate that there is a clear age-metallicity relation in the cluster with the younger stars being more metal-rich.

\section{Discussion}

\subsection{Age Range}

We have shown using several different methods that there is a difference in ages within the stars of $\omega$ Cen of between 2 and 4 Gyrs. Our first method of assigning individual ages to stars initially seemed to indicate quite a large age range of 5 Gyrs. It was found, however, through simulations of the populations in $\omega$ Cen that this large age range was most probably induced by the observational errors, which led to an enhanced age-metallicity correlation. Our simulations indicated that the actual age range in the cluster was $2 \pm 2$ Gyrs. Simulations of the CMDs, using on the photometry information, showed that the age range is $4 \pm 2$ Gyrs.

Although our results do not give a definitive value for the age range in $\omega$ Cen, they can be used, along with other data, to strongly constrain it. Previous results for the existence of an age range in $\omega$ Cen, and the method employed, are summarized in Table 5. Considering these studies, an age range of $\geq 6$ Gyrs is most likely to be too high. On the other hand, although a zero age range can not be ruled out completely, it seems unlikely to be the case, particularly given the results for the element abundance ratios in the metal-rich populations. We therefore conclude that the most likely value for the age spread in $\omega$ Cen is 2-4 Gyrs.

Two results in the literature are most relevant to this work. The first is that of Hilker et al. (2004). They used Strömgren photometry and metallicities to determine an age range of 3 Gyrs. Their result is consistent to what we found here.

The second result is from Sollima et al. (2005b) using two sets of photometry, and metallicities derived from spectra. They find little, if any, age range (0-2 Gyrs) in their isochrone fits to the CMDs. As we have found an age range of 2-4 Gyrs these results are not entirely inconsistent, but do differ enough to warrant further investigation. Part of the explanation for this difference may come from the two different regions of the cluster that we and Sollima et al. (2005b) have observed. Our photometric data come from the outer regions of the cluster between 15 and 25 arcminutes from the center, while the Sollima et al. (2005b) data

originated from fields centered on the cluster out to 10 arcminutes. The metal-rich population is more centrally concentrated (NFM96), and we may not be sampling enough of these 
objects to make a conclusive statement on the ages of the most metal-rich population. In apparent disagreement with there being no age range, however, is the lack of $\alpha$-enhancement in the most metal-rich population (Pancino et al. 2002) suggesting supernovae Ia involvement in the enrichment of the stars. Kobayashi et al. (1998) found the progenitors of supernovae Ia take $<1$ Gyr to evolve, which is in agreement with the result of Sollima et al. (2005b). However, Yoshii, Tsujimoto, \& Nomoto (1996) found that the lifetime of supernovae Ia progenitors is most likely to be 0.5-3 Gyrs, which supports both the result found here and Sollima et al. (2005b). An abundance study of the s-process elements by Pancino (2003) for the most metal-rich stars show enrichment on the same scales as the metal-intermediate population ([s/Fe] 1.0 dex) (Norris \& Da Costa 1995; Smith et al. 1995, 2000). The sources of these enrichment processes (AGB stars) take several gigayears to mature (Romano et al. 2005, and references therein).

Examining Figures 3 and 5 from Sollima et al. (2005b), which are relevant to their WFI data, one finds an age range of about 3 Gyrs is possible. Using isochrones with slightly different metallicities than those plotted by Sollima et al. (2005b) (for example $[\mathrm{Fe} / \mathrm{H}]=-$ 1.85 for the MP population and $[\mathrm{Fe} / \mathrm{H}]=-1.2$ for the MInt2 population, suggested by the mean abundance in the sample range determined from their Figure 3), ages of 17 Gyrs and 14 Gyrs are required to fit the MP and MInt2 data, respectively, giving an age range of 3 Gyrs. Further, the outliers in the right panel of their Figure 5, which are explained by Sollima et al. (2005b) as possibly due to photometric or spectroscopic errors, may instead require a much younger age. It would be interesting to know how far from cluster center these objects lie. A possible conclusion might be that there is a bimodality in age in the most metal-rich population where the older metal-rich group resides primarily in the center of the cluster, while the younger metal-rich group resides in the outer regions. Given the metallicity errors in both Sollima et al. (2005b) and our data sets, it is not possible to obtain a definitive answer to this possibility.

In Figures 4 and 6 of Sollima et al. (2005b), which pertain to a different, higher resolution data set, one does not find a similar age difference, except to say there is a large range in metallicities (shown in the upper panels of their Figure 4) for each population. This might indicate that there is a range of ages in each population. The lack of spread in the CMD for each metallicity group, however, suggest that the single metallicity isochrone fits to these data are an appropriate choice.

Throughout this work we have assumed the age-metallicity relation to be a linear one. This may not be the case. We know that $\omega$ Cen has at least three (and possibly up to five) distinct populations. The length of time between the formation of these populations may not in fact be linear. Unfortunately our data do not have the required accuracy to address 
this question. More accurate metallicities are required, for which higher resolution spectra are needed, as well as larger samples of the most metal-rich population, to more accurately determine the age range in the cluster.

\subsection{Cluster Origins}

The origin of $\omega$ Cen is not well understood. Due to its unique metallicity and age ranges it is unlikely that it formed in the same manner as other globular clusters. From the enrichment of its member stars, it was massive enough to retain ejecta from AGB stars and supernovae. Tsujimoto \& Shigeyama (2003) discuss the formation of globular clusters as the result of cloud-cloud collisions. These collisions trigger star formation, and chemical evolution in the resulting cluster depends on the relative velocity of the initial clouds. Those with low velocities trigger star formation involving less than $1 \%$ of the gas and promote star formation episodes induced by supernovae, as would be the case in $\omega$ Cen. On the other hand, collisions between clouds with higher velocities do not retain enough gas to form later generations after the initial star formation episode, resulting in "normal" globular clusters.

Alternatively, $\omega$ Cen may be the result of mergers of several globular clusters with discrete metallicities within the halo of the Milky Way. However, several globular clusters of discrete metallicites do not accurately reproduce the metallicity distribution seen on the RGB (NFM96, Smith et al. 2000), and the probability of several clusters colliding and merging in the halo is low. This scenario is also not consistent with the s-process enhancements seen in the more metal-rich populations. A different twist to this hypothesis is the merger within a fragment scenario (Searle 1977; Searle \& Zinn 1978). In the context of $\omega$ Cen, the more metal-rich component may have been another smaller cluster associated with the parent dwarf galaxy that merged with the nucleus (Norris et al. 1997; Ferraro et al. 2002).

Current evidence suggests $\omega$ Cen is most likely to be the remnant nucleus of a dwarf spheroidal galaxy that was consumed by the Milky Way (Freeman 1993), similar to the Sagittarius dwarf spheroidal galaxy (dSph), which is currently in the process of being stripped. The similarities between $\omega$ Cen and dSph galaxies was noted initially by Norris \& Bessell (1978). $\omega$ Cen shows self-enrichment over a timescale of several gigayears, consistent with this scenario. The current orbit of $\omega$ Cen within the Galaxy is at odds with cluster enrichment over a long timescale, suggesting that this is not where it initially formed. In its current orbit, the Milky Way would not have allowed such self-enrichment to take place since any gas would have been stripped from the cluster not long after its ejection. The retrograde motion and small apocentric radius (Dinescu et al. 1999a) are unusual properties for a globular cluster and further the suggestion that it did not originally form in its current orbit. Frequent 
disk crossings would also have stripped remaining gas from the cluster thereby stopping any later star formation episodes.

Bekki \& Freeman (2003) have demonstrated using a self-consistent dynamical model that $\omega$ Cen could have been formed from a nucleated dwarf galaxy that interacted and merged with the young Galactic disc over a period of 2.6 Gyrs. This model assumes that there is very little gas in the Galactic disc at these times. The central nucleus survives tidal stripping due to its compactness, and extended star formation is induced by the Galactic tidal forces causing radial inflow which triggers repetitive starbursts. Their Figure 4 shows that star formation is enhanced slightly at several different epochs. This is consistent with the age range found here for $\omega$ Cen and the distinct populations found in the cluster.

Numerical simulations have been used to analyze the dynamical evolution of simulated dwarf galaxies that evolve to have the present day kinematic characteristics of $\omega$ Cen (Mizutani, Chiba \& Sakamoto 2003; Chiba \& Mizutani 2004; Meza et al. 2005). As the disruption occurs, these systems may deposit large fractions of their stars into the thick disc component of the Galaxy, leaving the nucleus to orbit it. As Mizutani, Chiba \& Sakamoto (2003) note, the debris from the progenitor may have already been found in the observations showing signatures of merging events in the Milky Way (Gilmore, Wyse \& Norris 2002). Analysis of data from various surveys (such as the Sloan Digital Sky Survey (York et al. 2000) or RAdial Velocity Experiment (Steinmetz 2003)) may find the debris from the progenitor of $\omega$ Cen in the thick disc, giving a more comprehensive picture of the evolution of the cluster.

Abundance studies of the Sagittarius dwarf spheroidal galaxy have shown similar patterns to those found in $\omega$ Cen for some elements (McWilliam \& Smecker-Hane 2005a,b), further supporting the idea that the cluster was associated with an accreted dwarf galaxy. Deficiencies in copper are seen in both systems (McWilliam \& Smecker-Hane 2005a; Cunha et al. 2002; Pancino et al. 2002), and abundance patterns of other elements (La and Y) were also found to be similar. $[\alpha / \mathrm{Fe}], \mathrm{Na}$ and $\mathrm{Al}$, however, exhibit different patterns. This last result indicates that although both systems share a similar history, as one might expect, evolutionary differences exist between them. Care should be taken, however, when comparing $\omega$ Cen with dSph galaxies, as the relative sizes between the two systems are quite different. Despite this, comparisons can still be made between the systems as $\omega$ Cen, as the nucleus of a dSph, may have had gas inflow from the parent dSph outer regions that was incorporated into later generations of stars (Bekki \& Norris 2006).

CMDs of other dwarf galaxies show episodic star formation episodes. The Carina and Fornax dSphs show large ranges in both metallicity and age (Mateo 1998, and references therein). The Carina dwarf spheroidal galaxy is known to have two, possibly three, episodes of star formation (Smecker-Hane et al. 1994; Hurley-Keller, Mateo \& Nemec 1998; Mon- 
elli et al. 2003). These results are qualitatively similar to those found for $\omega$ Cen — star formation over relatively large timescales, once again supporting the idea that this cluster is the nucleated remnant of a dSph galaxy.

Another property of $\omega$ Cen is the dependence of kinematics on abundance. Norris et al. (1997) showed that the metal-rich component in the cluster is centrally concentrated and has a lower velocity dispersion than the metal-poor population. Sollima et al. (2005b) not only find the metal-intermediate populations have a lower velocity dispersion than the metal-poor component, in agreement with Norris et al. (1997), but further find the most metal-rich population has a higher velocity dispersion than the metal-intermediate, but not as large as the metal-poor. The Sculptor dSph galaxy shows the similar characteristic (Tolstoy et al. 2004) of dependence of kinematics on abundance. This dSph has two distinct populations, one metal-rich $([\mathrm{Fe} / \mathrm{H}]=-1.4)$ and the other metal-poor $([\mathrm{Fe} / \mathrm{H}]=-2.0)$. The higher metallicity stars show lower velocity dispersion than the metal-poor component and are also more centrally concentrated, just as reported for $\omega$ Cen. Tolstoy et al. (2004), however, found no evidence of different systemic rotation between the two components. In contrast, in $\omega$ Cen the metal-poor component exhibits systemic rotation while the metal-rich one does not (Norris et al. 1997), showing that there are differences between the systems that are yet to be explained.

\section{Conclusions}

Interpretation of our age-metallicity diagram is complicated by correlated errors in metallicity and age, but after extensive simulations an age range of 2-4 Gyrs is found to exist in $\omega$ Cen. We find an age-metallicity relation where the younger stars are those that are more metal-rich. These results strengthen the likelihood that the origin of the unusual properties of this cluster is connected with the evolution of a more massive system such as a nucleated dwarf galaxy that was subsequently captured and disrupted by the Milky Way.

We thank the Director and staff of the Anglo Australian Observatory for the use of their facilities.

\section{REFERENCES}

Bedin, L. R., Piotto, G., Anderson, J., Cassisi, S., King, I. R., Momany, Y., \& Carraro, G. 2004, ApJ, 605, L125

Beers, T. C., Rossi, S., Norris, J. E., Ryan, S. G., \& Shefler, T. 1999, AJ, 117, 981 
Bekki, K., \& Freeman, K. C. 2003, MNRAS, 346, 11

Bekki, K., \& Norris, J. E. 2006, ApJ, 637, 109

Cannon, R. D., 1981, MNRAS, 195, 1

Cannon, R. D., \& Stewart, N. J. 1981, MNRAS, 195, 15

Cannon, R. D., \& Stobie, R. S. 1973a, MNRAS, 162, 207

Carney, B. W., Laird, J. B., Latham, D. V., \& Aguilar, L. A., 1996, AJ, 112, 668

Chiba, M., \& Mizutani, A. 2004, PASA, 21, 237

Cunha, K., Smith, V. V., Suntzeff, N. B., Norris, J. E., Da Costa, G. S., \& Plez, B. 2002, AJ, 124,379

Dinescu, D I., van Altena, W F., Girard, T M., \& Lpez, C E. 1999a, AJ, 117, 277

Ferraro, F. R., Bellazzini, M., \& Pancino, E. 2002, ApJ, 573, L95

Ferraro, F. R., Sollima, A., Pancino, E., Bellazzini, M., Straniero, O., Origlia, L., \& Cool, A. M. 2004, ApJ, 603, L81

Freeman, K. C. 1993, in Smith, G. H., \& Brodie, J. P., eds, ASP Conf. Ser. Vol. 48, The Globular Cluster-Galaxy Connection. (Astron. Soc. Pac.: San Francisco), p608

Freeman, K. C., \& Rodgers, A., W. 1975, ApJ, 201, L71

Freyhammer, L. M., Monelli, M., Bono, G., Cunti, P., Ferraro, I., Calamida, A., Degl'Innocenti, S., Prada Moroni, P. G., Del Principe, M., Piersimoni, A., Iannicola, G., Stetson, P. B., Andersen, M. I., Buonanno, R., Corsi, C. E., Dall'Ora, M., Petersen, J. O., Pulone, L., Sterken, C., \& Storm, J. 2005, ApJ, 623, 860

Gilmore, G., Wyse, R. F. G., \& Norris, J. E. 2002, ApJ, 574, L39

Green, E. M., Demarque, P., \& King, C. R. 1987, The Revised Yale Isochrones \& Luminosity Functions (New Haven: Yale Univ. Obs.)

Harris, W. E., 1996, AJ, 112, 1478

Hilker, M., Kayser, A., Richtler, T., \& Willemsen, P. 2004, A\&A, 442, L9

Hilker, M., \& Richtler, T. 2000, A\&A, 362, 895 
Hughes, J., \& Wallerstein, G. 2000, AJ, 119, 1225

Hurley-Keller, D., Mateo, M., \& Nemec, J. 1998, AJ, 115, 1840

Kim, Y., Demarque, P., Yi, S., \& Alexander, D. 2002, ApJS, 143, 499

Kobayashi, C, Tsugimoto, T., Nomoto, K., Hachisu, I., \& Kato, M. 1998, ApJ, 503, L159

Lee, Y.-W., Joo, J.-M., Sohn, Y.-J., Rey, S.-C., Lee, H.-C., Walker, A. R. 1999, Nature, 402, 55

Lee, Y.-W., Joo, S.-J., Han, S.-I., Chung, C., Ree, C. H., Sohn, Y.-J., Kim, Y.-C., Yoon, S.-J., Yi, S. K. \& Demarque, P. 2005, ApJ, 621, L57

Lee, Y.-W., Rey, S.-C., Ree, C. H., Joo, J. M., Sohn, Y.-J., Yoon, S.-J., \& Walker, A. 2002, eds, ASP Conf. Ser. Vol. 265, A Unique Window into Astrophysics. (Astron. Soc. Pac.: San Francisco), p. 305

Lewis et al., 2002, MNRAS, 333, 279

Lloyd Evans, T. 1997, MNRAS, 181, 591

Lub, J., 2002, in van Leeuwen, F., Hughes, J. D., \& Piotto, G. 2002, eds, ASP Conf. Ser. Vol. 265, A Unique Window into Astrophysics. (Astron. Soc. Pac.: San Francisco), p. 95

Mateo, M. 1998, ARA\&A, 36, 435

McWilliam, A., \& Smecker-Hane, T. A. 2005a, ApJ, 622, L29

McWilliam, A., \& Smecker-Hane, T. A. 2005b, eds, ASP Conf. Ser. Vol. 336, Cosmic Abundances as Records of Stellar Evolution and Nucleosynthesis in honor of David L. Lambert. (Astron. Soc. Pac.: San Francisco), p. 221

Merritt, D., Meylan, G., \& Mayor, M. 1997, AJ, 114, 107

Meza, A., Navarro, J. F., Abadi, M. G., \& Steinmetz, M. 2005, MNRAS, 359, 93

Mizutani, A., Chiba, M., \& Sakamoto, T. 2003, ApJ, 589, L89

Monelli, M., Pulone, L., Corsi, C. E., Casterllani, M., Bono, G., Waker, A. R., Brocato, E., Buonanno, R., Caputo, F., Castellani, V., Dall'Ora, M., Marconi, M., Nonino, M., Ripepi, V., \& Smith, H. A. 2003, AJ, 126, 218

Norris, J. E. 2004, ApJ, 612, L25 
Norris, J., \& Bessell, M. S. 1978, ApJ, 225, L49

Norris, J. E., \& Da Costa, G. S. 1995, ApJ, 447, 680

Norris, J. E., Freeman, K. C., Mayor, M., \& Seitzer, P. 1997, ApJ, 487, L187

Norris, J. E., Freeman, K. C., \& Mighell, K. J. 1996, ApJ, 462, 241

Origlia, L., Ferraro, F. R., Bellazzini, M., \& Pancino, E. 2003, ApJ, 591, 916

Pancino, E. 2003, Multiple Stellar Populations in $\omega$ Centauri, PhD thesis, University of Bologna, Italy

Pancino, E., Ferraro, F. R., Bellazzini, M., Piotto, G., \& Zoccali, M. 2000, ApJ, 534, 83

Pancino, E., Pasquini, L., Hill, V., Ferraro, F. R., Bellazzini, M. 2002, ApJ, 568, L101

Piotto, G., Villanova, S., Bedin, L. R., Gratton, R., Cassisi, S., Momany, Y., Recio-Blanco, A., Lucatello, S., Anderson, J., King, I. R., Peitrinferni, A. \& Carraro, G. 2005, ApJ, 621,777

Rey, S.-C., Lee, Y.-W., Ree, C. H., Joo, M.-J., \& Sohn, Y.-J. 2004, AJ, 127, 958

Romano, D., Chiappini, C., Matteucci, F., \& Tosi, M. 2005, å, 430, 491

Searle, L. 1977, in Tinsley, B. M., \& Larson, R. B. 1977, eds. The Evolution of Galaxies and Stellar Populations. Yale Univ. Obs., New Haven, p.219

Searle, L. \& Zinn, R. 1978, ApJ, 225, 357

Smecker-Hane, T. A., Stetson, P. B., Hesser, J. E., \& Lehnert, M. D. 1994, AJ, 108, 507

Smith, V., Cunha, K., \& Lambert, D. 1995, AJ, 110, 2827

Smith, V., Suntzeff, N., Cunha, K., Gallino, R., Busso, M., Lambert, D., \& Straniero, O. 2000, AJ, 119, 1239

Sollima, A., Ferraro, F. R., Pancino, E. \& Bellazzini, M. 2005, MNRAS, 357, 265

Sollima, A., Pancino, E., Ferraro, F. R., Bellazzini, M., Straniero, O., Pasquini, L. 2005, ApJ, 634, 332

Stanford, L. M, Da Costa, G. S., Norris, J. E., Cannon, R. D. 2004, Mem. Soc. Astron. Italiana, 75, 290 
Steinmetz, M. 2003, in GAIA Spectroscopy: Science and Technology, ASP Conf. Proc., 298, ed. U. Munari, 381

Suntzeff, N. B., \& Kraft, R., P. 1996, AJ, 111, 1913

Tolstoy, E., Irwin, M. J., Helmi, A., Battaglia, G., Jablonka, P., Hill, V., Venn, K. A., Shetrone, M. D., Letarte, B., Cole, A. A., Primas, F., Francoise, P., Arimoto, N., Sadakane, K., Kaufer, A., Szeifert, T., \& Abel, T. 2004, ApJ, 617, L119

Tsujimoto, T., \& Shigeyama, T. 2003, ApJ, 2003, 590, 803

Woolley, R. v. d. R., et al. 1996, R. Obs. Ann., No 2

Yi, S., Demarque, P., Kim, Y.-C., Lee, Y.-W., Ree, C.-H., Lejeune, T., \& Barnes, S. 2001, ApJ, 136, 417

York, D. G. et al. 2000, AJ, 120, 1579

Yoshii, Y., Tsujimoto, T., \& Nomoto, K. 1996, ApJ, 462, 266 
Table 1: Photometry errors for the $\omega$ Cen data.

\begin{tabular}{ccc}
\hline \hline$V$ mag & err $V$ & $\operatorname{err}(B-V)$ \\
\hline 16.0 & 0.002 & 0.004 \\
16.5 & 0.002 & 0.005 \\
17.0 & 0.004 & 0.007 \\
17.5 & 0.005 & 0.009 \\
18.0 & 0.008 & 0.012 \\
18.5 & 0.011 & 0.016 \\
19.0 & 0.017 & 0.025 \\
19.5 & 0.024 & 0.034 \\
20.0 & 0.031 & 0.045 \\
\hline \hline
\end{tabular}


Table 2. $\omega$ Cen Members. The full version of this table is available electronically.

\begin{tabular}{ccccccccccc}
\hline \hline ID & $\begin{array}{c}\text { RA } \\
\text { J2000 }\end{array}$ & $\begin{array}{c}\text { Dec } \\
\text { J2000 }\end{array}$ & Vmag & $\begin{array}{c}\mathrm{B}-\mathrm{V} \\
\mathrm{kms}^{-1}\end{array}$ & $\begin{array}{c}\mathrm{Vel} / \mathrm{H}] \\
\mathrm{F}_{[\mathrm{Fe} / \mathrm{H}]}\end{array}$ & $\begin{array}{c}\text { Age } \\
(\mathrm{Gyrs})\end{array}$ & $\begin{array}{c}\sigma_{\text {Age }} \\
(\mathrm{Gyrs})\end{array}$ & Run $^{1}$ \\
\hline 1000258 & 132549.30 & -471535.1 & 18.49 & 0.52 & 200 & -1.76 & 0.19 & 12.8 & 2.7 & 1 \\
1000812 & 132547.00 & -471723.0 & 17.47 & 0.61 & 227 & -1.64 & 0.19 & 12.3 & 1.3 & 2 \\
1002064 & 132541.80 & -471839.6 & 18.29 & 0.57 & 197 & -1.41 & 0.17 & 14.7 & 2.1 & 1 \\
1002884 & 132538.00 & -471946.6 & 17.49 & 0.60 & 206 & -1.76 & 0.19 & 12.8 & 1.2 & 2 \\
1004374 & 132531.00 & -471924.9 & 17.50 & 0.62 & 212 & -1.72 & 0.19 & 13.0 & 1.1 & 2 \\
1005088 & 132527.60 & -471333.8 & 17.39 & 0.73 & 224 & -1.58 & 0.22 & 17.5 & 3.9 & 2 \\
1005184 & 132526.70 & -471950.2 & 17.28 & 0.72 & 225 & -1.93 & 0.30 & 19.0 & 2.1 & 2 \\
1005758 & 132523.70 & -471726.6 & 17.38 & 0.80 & 211 & -1.37 & 0.28 & 19.0 & 3.2 & 2 \\
1005996 & 132521.80 & -472548.1 & 17.32 & 0.72 & 223 & -1.49 & 0.24 & 12.4 & 4.3 & 2 \\
1006065 & 132522.30 & -471209.1 & 17.51 & 0.65 & 226 & -1.62 & 0.20 & 13.0 & 1.6 & 2 \\
\hline
\end{tabular}

${ }^{1}$ Observed in 98/99 (1), $2002(2)$ 
Table 3. Non-Members in the 2002 observing box. The full version of this table is available electronically.

\begin{tabular}{cccccrr}
\hline \hline ID & $\begin{array}{c}\text { RA } \\
\text { J2000 }\end{array}$ & $\begin{array}{c}\text { Dec } \\
\text { J2000 }\end{array}$ & Vmag & $\begin{array}{c}\text { B-V } \\
\text { Vel }\end{array}$ & $\begin{array}{l}\text { Run }^{1} \\
\mathrm{~km} \mathrm{~s}^{-1}\end{array}$ \\
\hline 1001938 & 132847.01 & -473448.40 & 17.960 & 0.745 & 83.82 & 2 \\
1002364 & 132820.23 & -472721.50 & 17.454 & 0.935 & -69.53 & 2 \\
1004333 & 132831.77 & -471956.80 & 17.256 & 0.988 & -69.60 & 2 \\
1006419 & 132652.11 & -471147.70 & 17.963 & 0.957 & 19.28 & 2 \\
1006806 & 132656.74 & -470501.60 & 17.773 & 0.725 & 51.16 & 2 \\
1006842 & 132640.69 & -470541.80 & 17.567 & 0.785 & 2.03 & 2 \\
1007176 & 132602.32 & -470748.90 & 18.112 & 0.979 & -5.43 & 2 \\
1007243 & 132526.06 & -471303.70 & 18.387 & 0.725 & -36.33 & 2 \\
1007513 & 132459.11 & -471947.20 & 17.850 & 0.931 & -15.36 & 2 \\
1008138 & 132453.74 & -472640.90 & 17.625 & 0.828 & -66.73 & 2 \\
\hline
\end{tabular}

${ }^{1}$ Observed in 98/99 (1), $2002(2)$ 
Table 4: Parameters and observing dates for the calibrating clusters.

\begin{tabular}{lccccc}
\hline Cluster & $\mathrm{E}(B-V)$ & $(\mathrm{m}-\mathrm{M})_{V}$ & {$[\mathrm{Fe} / \mathrm{H}]$} & Number & Observed \\
\hline NGC 6397 & 0.17 & 12.36 & -1.95 & 111 & 23 Jul 98 \\
M 55 & 0.10 & 13.87 & -1.81 & 70 & 23 Jul 98 \\
& & & & & 04 Jul 00 \\
NGC 6752 & 0.05 & 13.13 & -1.56 & 114 & 22 Sep 98 \\
& & & & & 16 May 99 \\
& & & & & 17 May 99 \\
47 Tuc & 0.04 & 12.37 & -0.76 & 147 & 22 Nov 00 \\
& & & & & 23 Nov 00 \\
\hline
\end{tabular}


Table 5: Age Ranges in the Literature

\begin{tabular}{|c|c|c|}
\hline Reference & Method & Age Range \\
\hline \multirow[t]{2}{*}{ Norris \& Da Costa (1995) } & Spect. of RGB stars & \\
\hline & s-process enrichment & $\geq 1 \mathrm{Gyr}$ \\
\hline Hilker \& Richtler (2000) & Strömgren photometry & 3-6 Gyrs \\
\hline Hughes \& Wallerstein (2000) & Strömgren photometry & $\geq 3$ Gyrs \\
\hline Smith et al. (2000) & Spect. of RGB stars & 2-3 Gyrs \\
\hline Lee et al. (2002) & Photometry, Red clump HB & 4 Gyrs \\
\hline \multirow[t]{2}{*}{ Pancino et al. (2002) } & Spect. of RGB stars & \\
\hline & SNe I enrichment & $\leq 1 \mathrm{Gyr}$ \\
\hline \multirow[t]{2}{*}{ Origlia et al. (2003) } & Spect. of RGB stars & \\
\hline & SNe I enrichment & $\sim 1 \mathrm{Gyr}$ \\
\hline \multirow[t]{2}{*}{ Ferraro et al. (2004) } & High res. multiband phot. & \\
\hline & Subgiant isochrone fitting & 0 Gyrs \\
\hline \multirow[t]{2}{*}{ Hilker et al. (2004) } & Spect. \& photometry & \\
\hline & of MSTO stars & 3 Gyrs \\
\hline \multirow[t]{2}{*}{ Rey et al. (2004) } & BV, Ca, Strömgren phot. & \\
\hline & Population models of HB & 4 Gyrs \\
\hline Sollima et al. (2005a) & RGB bumps & $<6$ Gyrs \\
\hline \multirow[t]{2}{*}{ Sollima et al. (2005b) } & SGB metallicities and & \\
\hline & isochrone fitting & $0-2$ Gyrs \\
\hline \multirow[t]{2}{*}{ This Paper } & Spectroscopy, photometry & \\
\hline & \& simulations of MSTO stars & 2-4 Gyrs \\
\hline
\end{tabular}




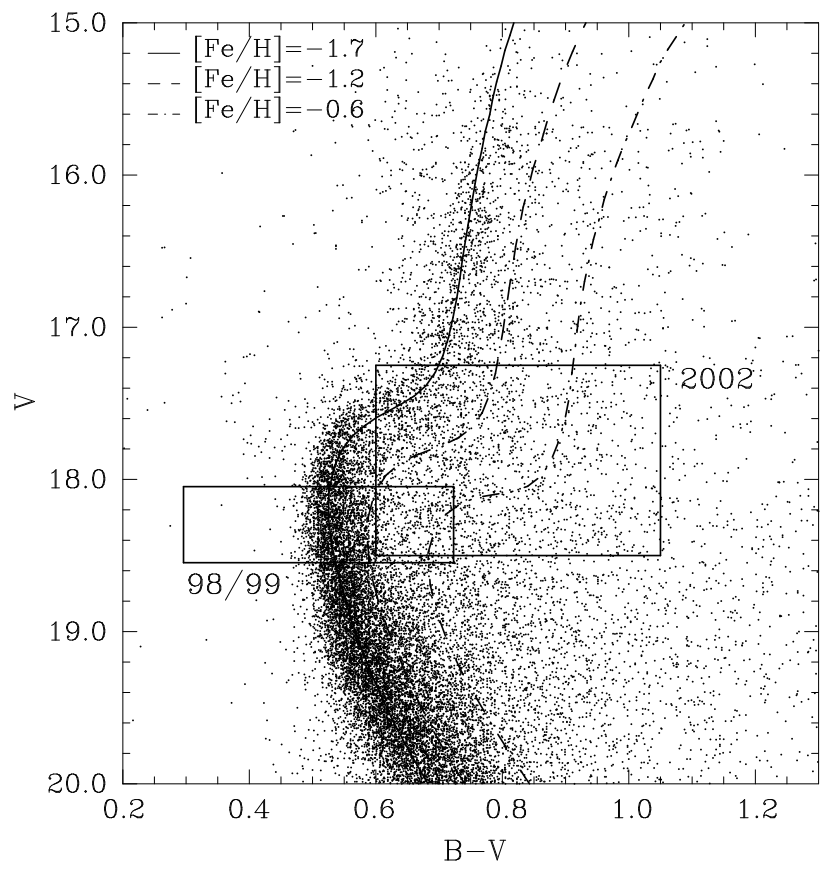

Fig. 1. - Color magnitude diagram for $\omega$ Cen. The isochrones (Yi et al. 2001) are for (left to right) $[\mathrm{Fe} / \mathrm{H}]=-1.7,-1.2$, and -0.6 . Each isochrone has an age of 13.5 Gyrs. The two boxes represent the areas that were used to find potential candidates from which to obtain spectra of cluster members. 


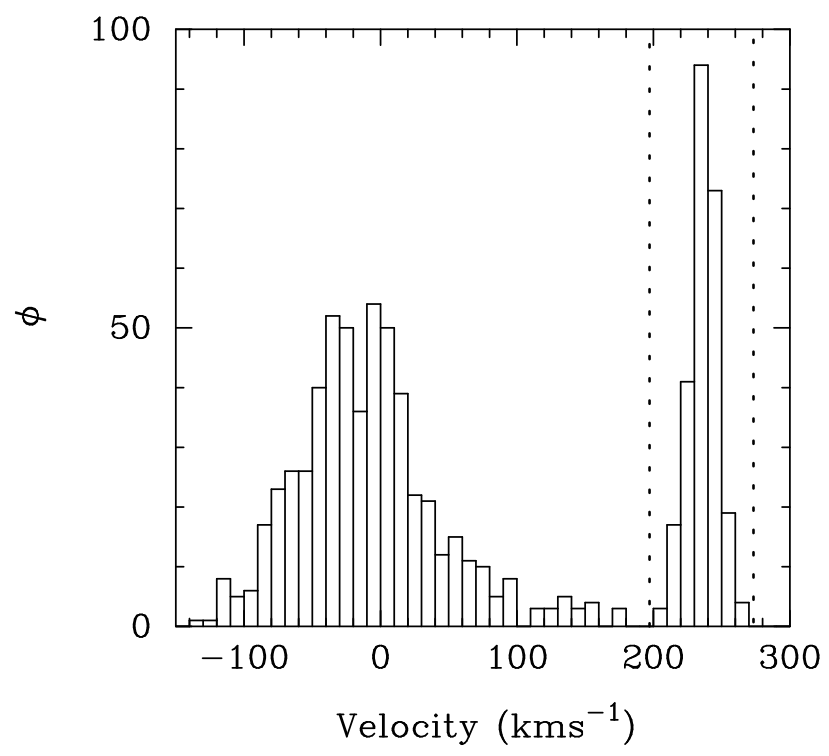

Fig. 2.- Histogram showing the velocities obtain for all the stars observed in 2002. The peak at $235 \mathrm{kms}^{-1}$ comprises the $\omega$ Cen members. The two vertical lines represent the $\pm 3 \sigma$ $\left(\sigma=13 \mathrm{kms}^{-1}\right)$ cutoff limits applied for membership classification. 


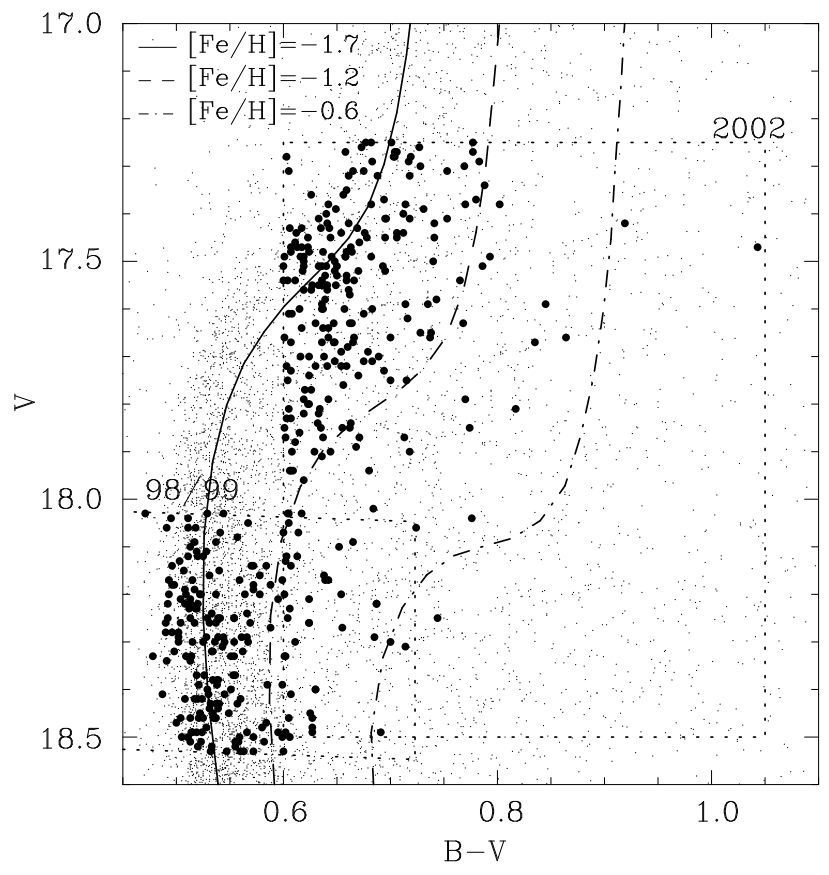

Fig. 3.- Color magnitude diagram for the members found in the two observed samples, represented by large dots. The small dots are plotted to show where the bulk of the stars from the photometry lie. These have no membership information. The isochrones are for $[\mathrm{Fe} / \mathrm{H}]=-1.7,-1.2,-0.6$ and each has an age of 13.5 Gyrs. 


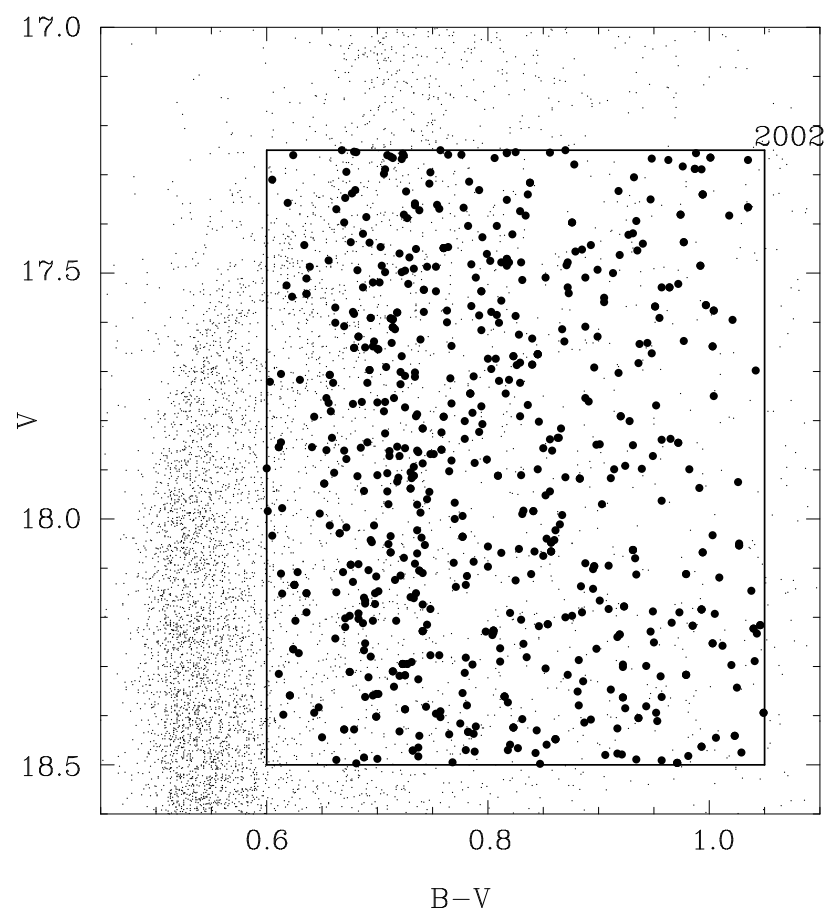

Fig. 4.- Color magnitude diagram for the non-members in the 2002 observing run. 


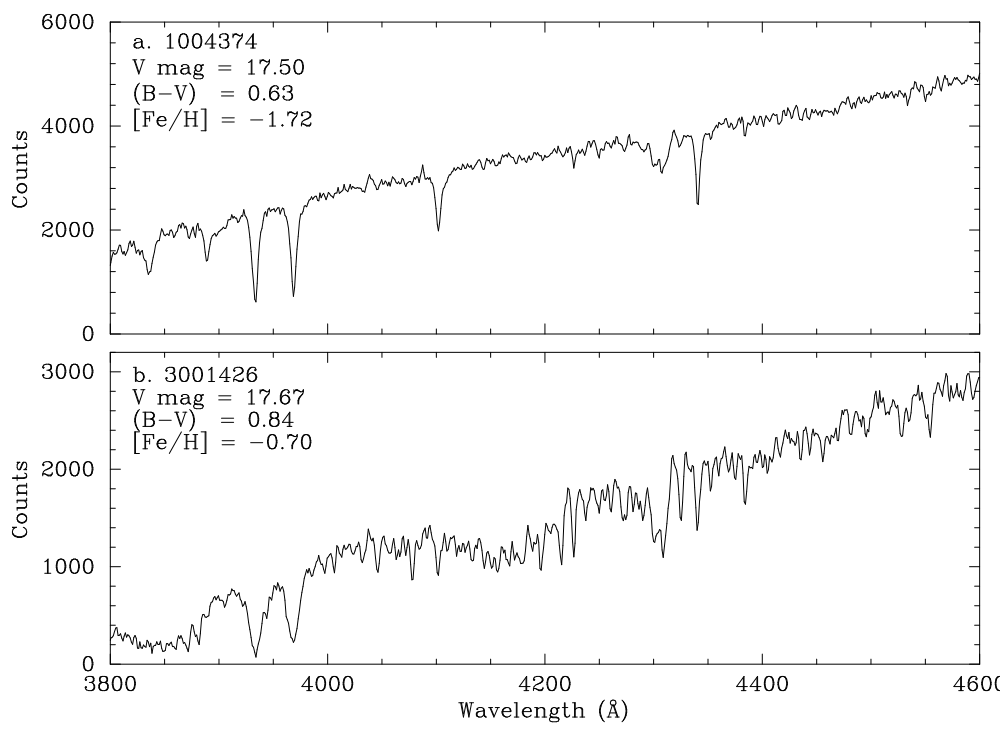

Fig. 5.- Spectra of two stars in our sample. The first, in the top panel, is a subgiant from the metal-poor population. The second is of a subgiant from one of the more metalrich populations. These spectra have a resolution of $\sim 2.4 \AA$. Noticeable differences are the increased $\mathrm{G}$ band at $\sim 4300 \AA, \mathrm{CN}$ at $3883 \AA$ and $4215 \AA$ and stronger metal lines in the more metal-rich star. 

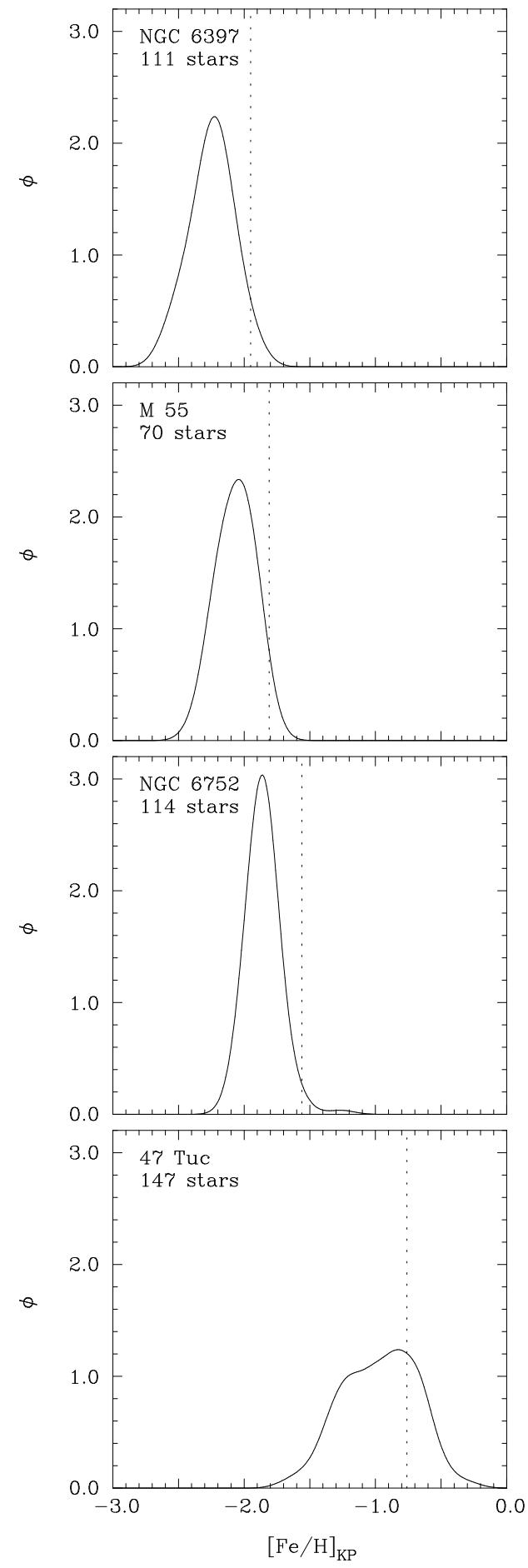

Fig. 6. - $\mathrm{K}^{\prime}$ metallicity generalized histograms for the members of the calibrating clusters NGC 6397, M55, NGC 6752 and 47 Tuc. The dotted line in each panel indicates the accepted $[\mathrm{Fe} / \mathrm{H}]$ value (Harris 1996), which is consistently of higher metallicity than the mean $[\mathrm{Fe} / \mathrm{H}]$ determined here. 


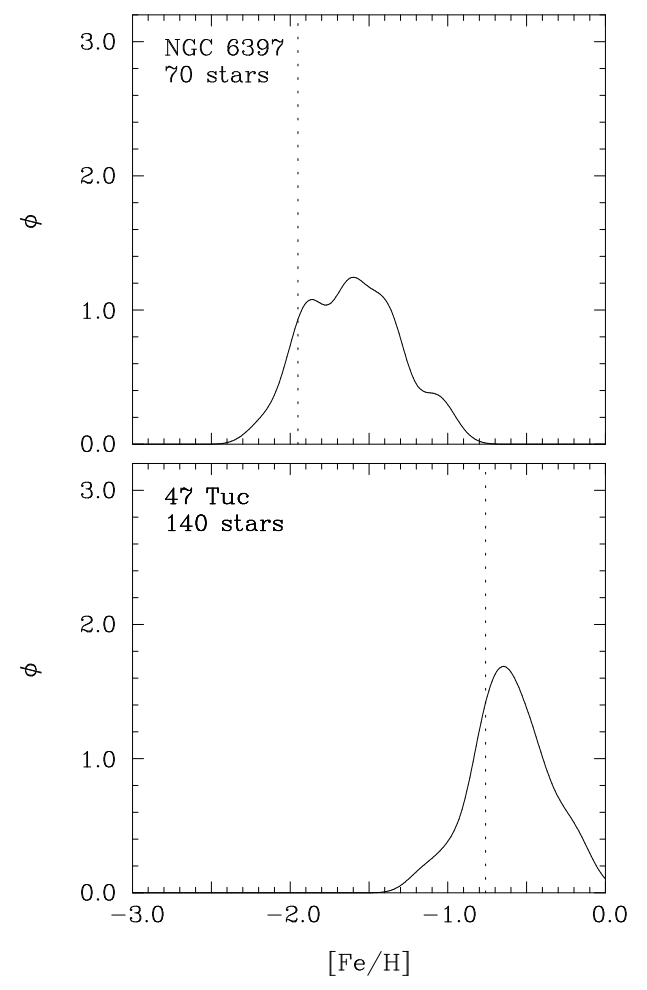

Fig. 7.- ACF metallicity histograms for NGC 6397 and 47 Tuc. Again, the dotted line shows the accepted $[\mathrm{Fe} / \mathrm{H}]$ for the cluster. Note that this line is more metal-poor than the mean of each distribution. 


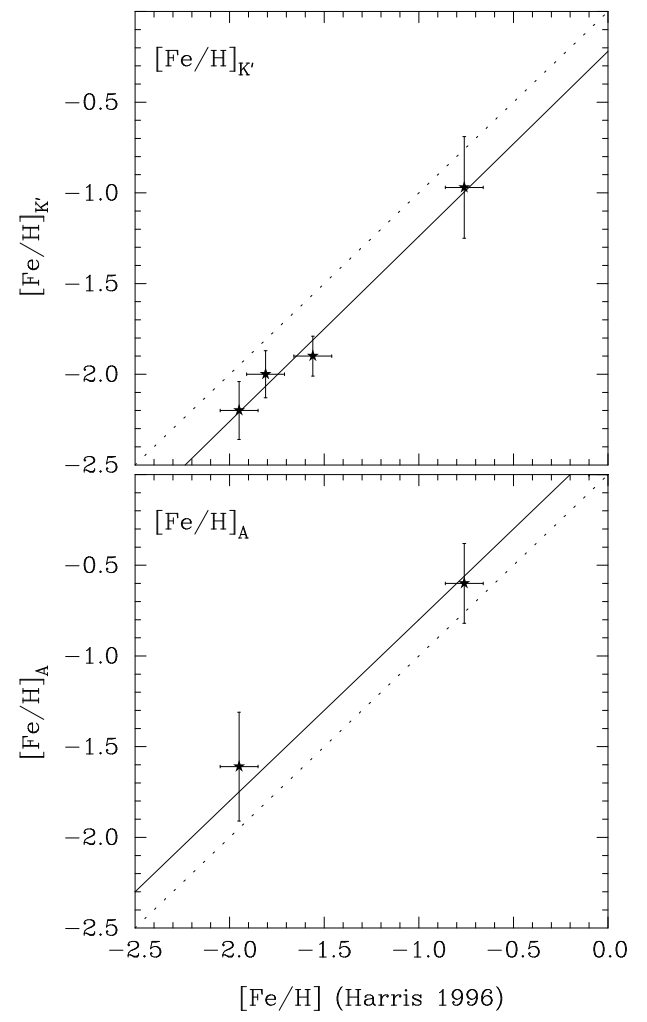

Fig. 8.- Comparison of the mean $[\mathrm{Fe} / \mathrm{H}]$ determined from the cluster metallicity distributions with the accepted $[\mathrm{Fe} / \mathrm{H}]$ (Harris 1996). The upper panel is for the $\mathrm{K}^{\prime}$ calibration, while the bottom shows the results from the ACF calibration. The dotted line in each panel indicates the 1:1 line. The solid line in the upper panel is the least-squares fit to the data, and in the lower panel represents the 0.2 dex offset to the 1:1 line. 


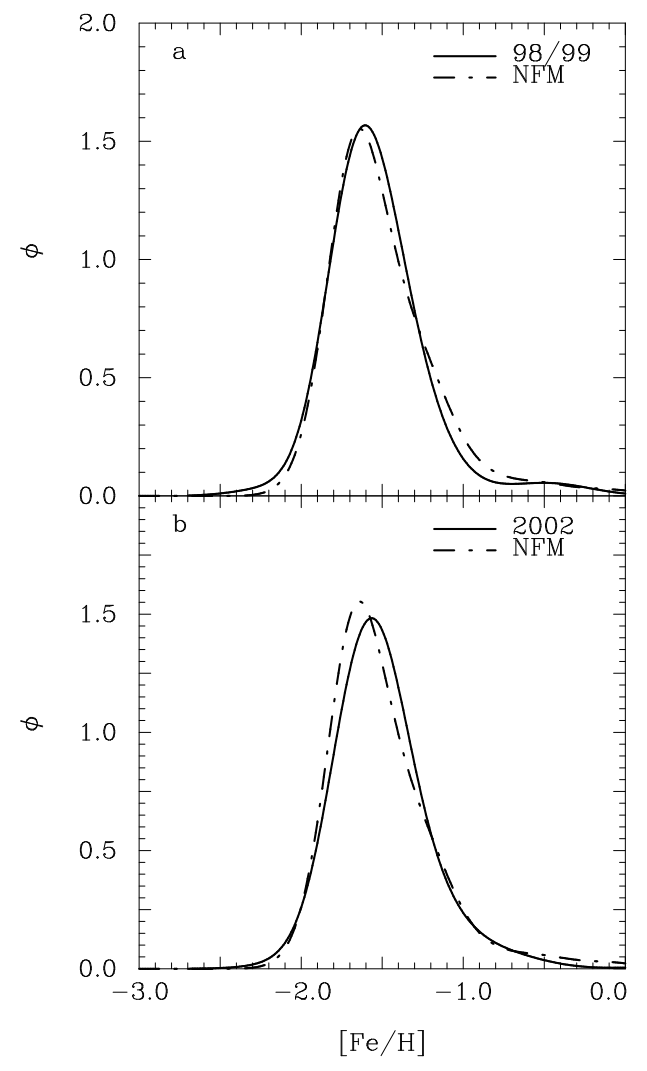

Fig. 9.- Generalized histograms of the metallicity distribution for the 98/99 (panel a) and 2002 (panel b) samples (gaussian kernal of $\sigma=0.15-0.20$, based on the individual errors for each metallicity). For comparison, the Norris, Freeman \& Mighell (1996) red giant branch distribution is also plotted $(\sigma=0.14)$. 

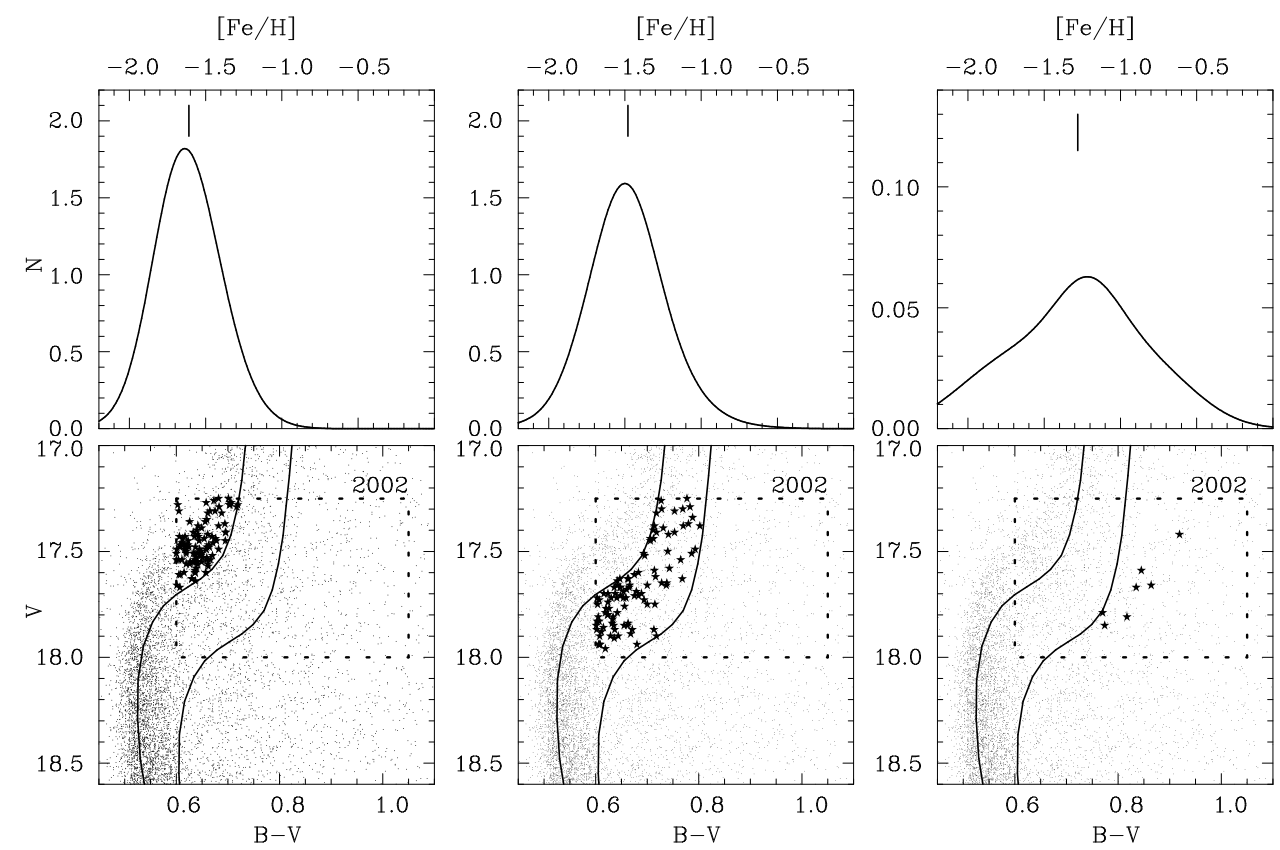

Fig. 10.- CMD split into three different areas, designated by the solid lines which were isochrones of the two metal-poor populations but shifted in color and luminosity. Generalized histograms were constructed using the data for the corresponding groups. The histograms show the metallicity distributions for those areas on the CMDs. 


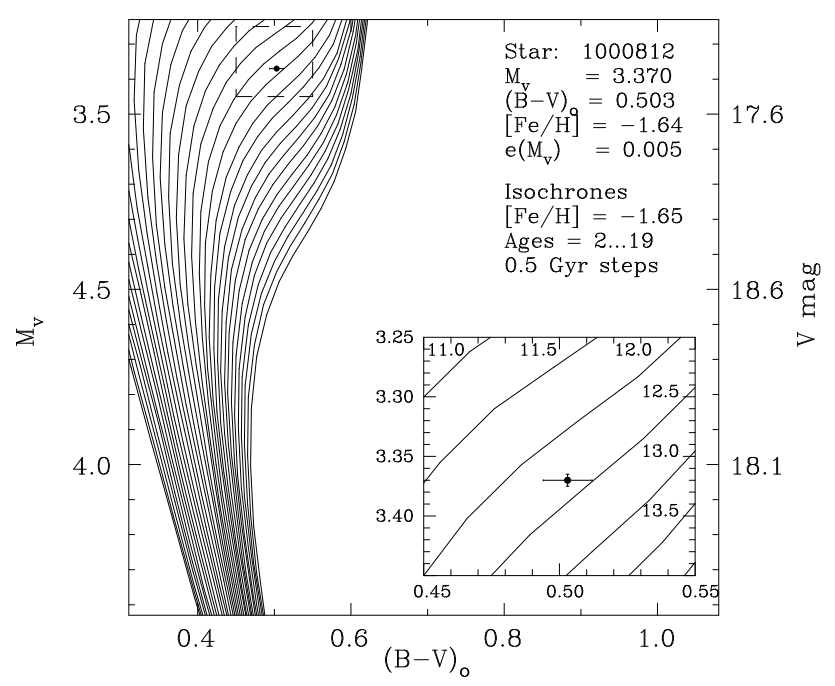

Fig. 11.- Age-metallicity diagram for the $\omega$ Cen sample, showing only the turnoff stars, as this is the area of the CMD that is most sensitive to age. 


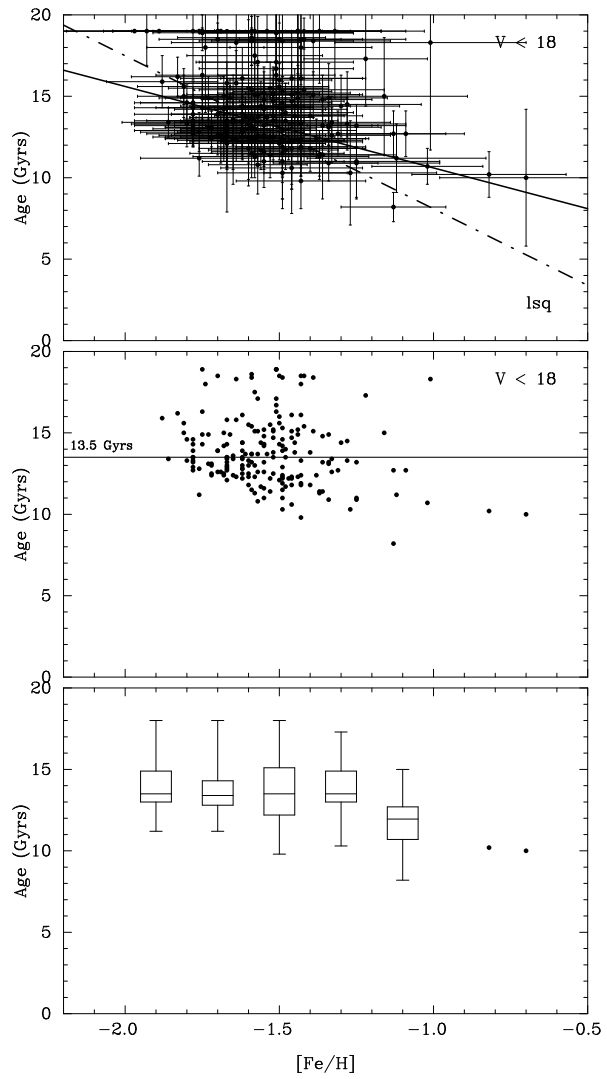

Fig. 12.- Age-metallicity diagrams for the simulations. Panel a shows the simulation with an input age of 0 Gyrs. Panels b, c and d show simulations with input ages of 2, 4 and 6 Gyrs, respectively. The input metallicity range comes from Norris, Freeman \& Mighell (1996). The dotted line in each panel represents the age-metallicity relation (Sim. AR) adopted for each simulation. The solid line is the least-squared fit to the data giving a calculated age range (Calc. AR). See text (§4.1) for more details. 

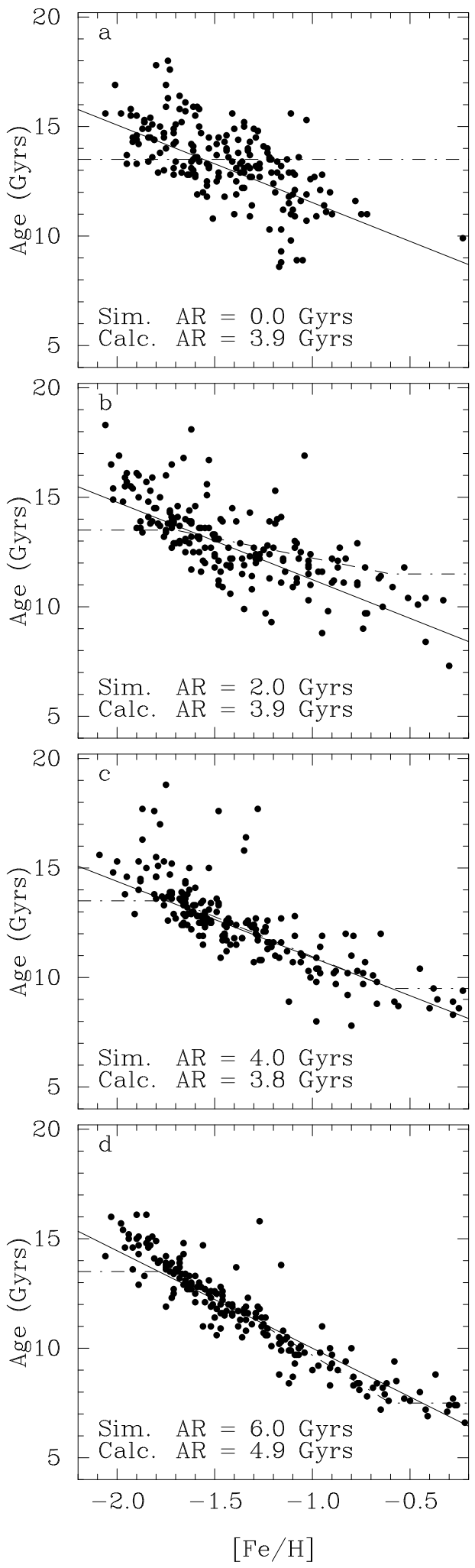

Fig. 13.- Observed age-metallicity diagram for $\omega$ Cen for comparison with the simulations in Figure 12. This is the same as Figure 11 without the error bars or 19 Gyr old stars. 


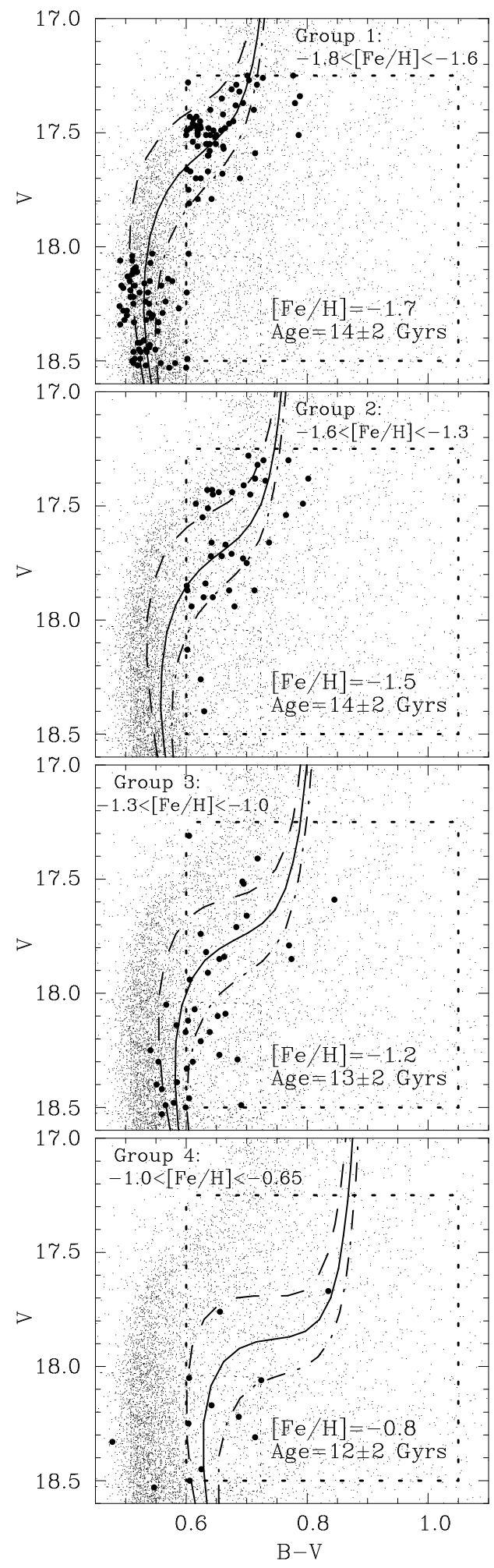

Fig. 14.- Metallicity cuts were made in the data, shown at the top of each panel, and isochrones with metallicities corresponding to the mean metallicity in each group were plotted with ages that best fit the data. This figure confirms the age range found in $§ 4.1$. 

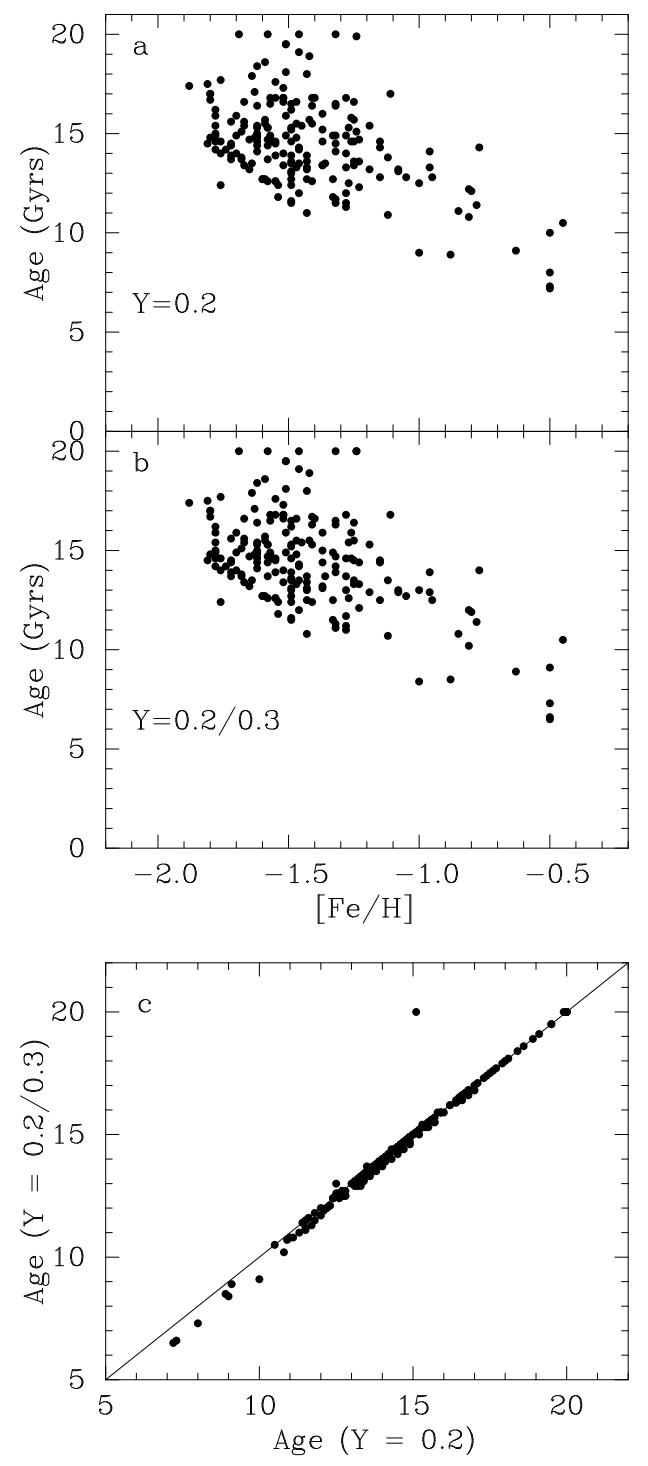

Fig. 15. - Age-metallicity relations resulting from ages obtained using constant He abundance $(Y=0.2)$ in panel a, and varied helium abundance $(\mathrm{Y}=0.2$ for $[\mathrm{Fe} / \mathrm{H}] \leq-1.5$ and 0.3 for $[\mathrm{Fe} / \mathrm{H}]>-1.5)$ in panel $\mathrm{b}$ for $V<18$. Panel c shows the comparison between the ages calculated using the two different sets of isochrones. See text (§4.2) for details. 


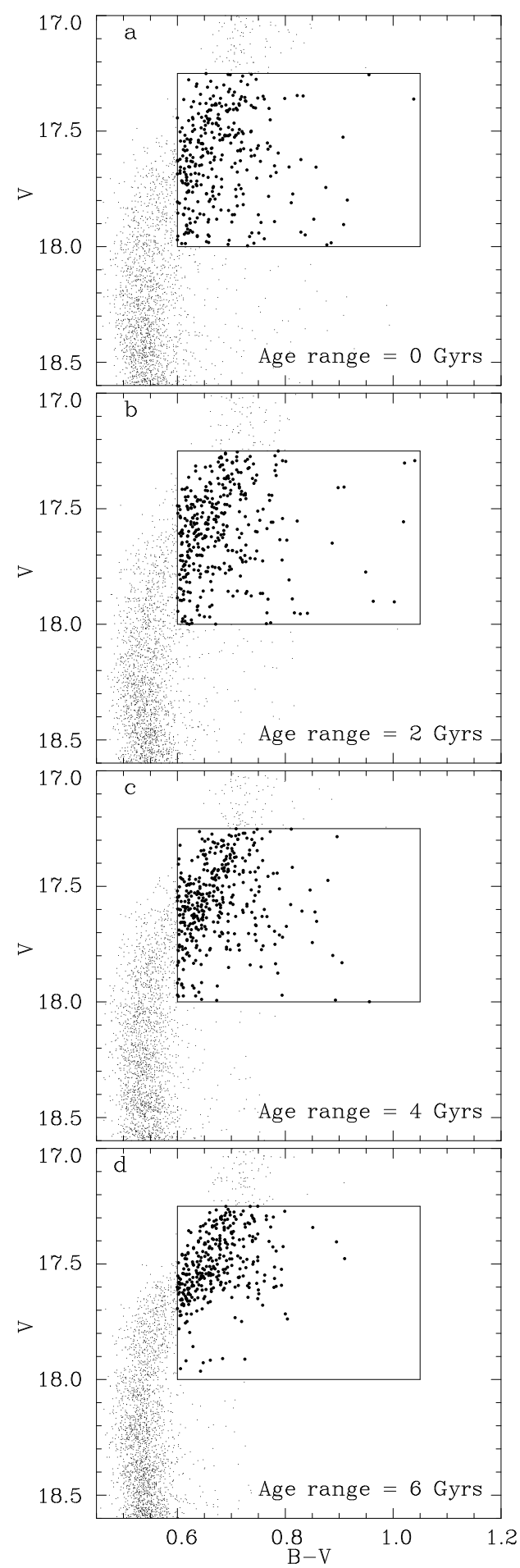

Fig. 16.- Examples of synthetic CMDs obtained from four simulations. See text (§4.3) for details. 


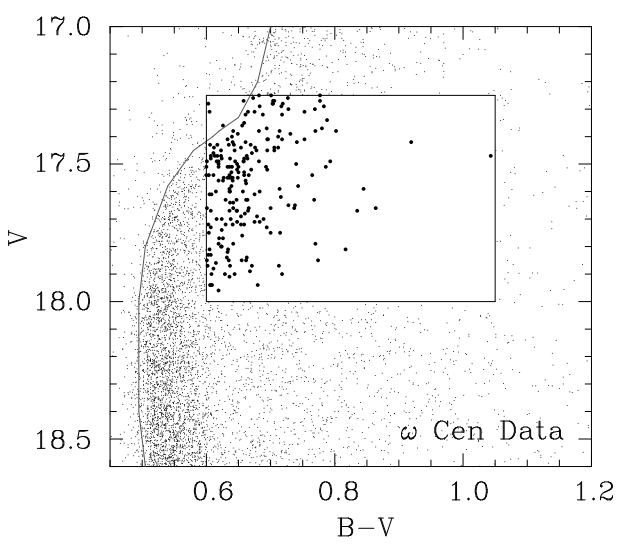

Fig. 17.- CMD for $\omega$ Cen for comparison with the synthetic simulations shown in Figure 16. The data plotted here are only for stars in our sample that have $V<18$. The solid line indicates the blue fiducial edge of the $\omega$ Cen data shown here, relevant to the discussion in $\S 4.4$ and the accompanying Figure 20. 

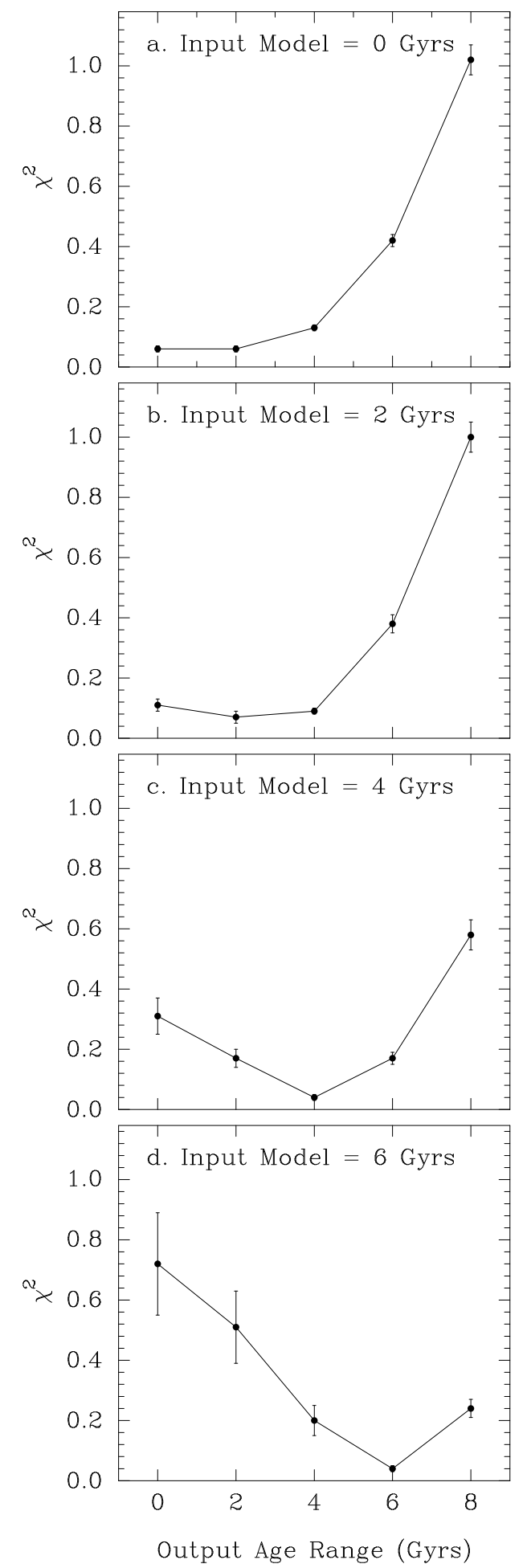

Fig. 18. - $\chi^{2}$ results for a set of representative samples with age ranges of 0, 2, 4 and 6 Gyrs tested against simulations of different age ranges. See text $(\S 4.3)$ for details. 


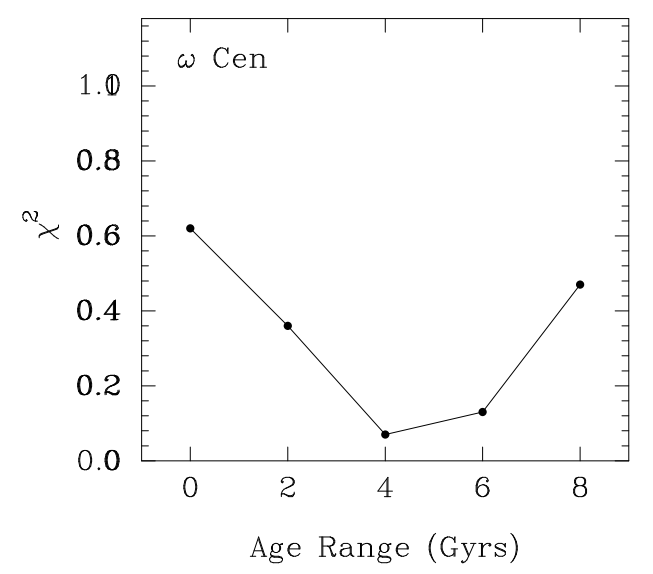

Fig. 19. $-\chi^{2}$ results for $\omega$ Cen. A comparison between this and Figure 18 shows that the age range in $\omega$ Cen is most likely to be 4 Gyrs. 


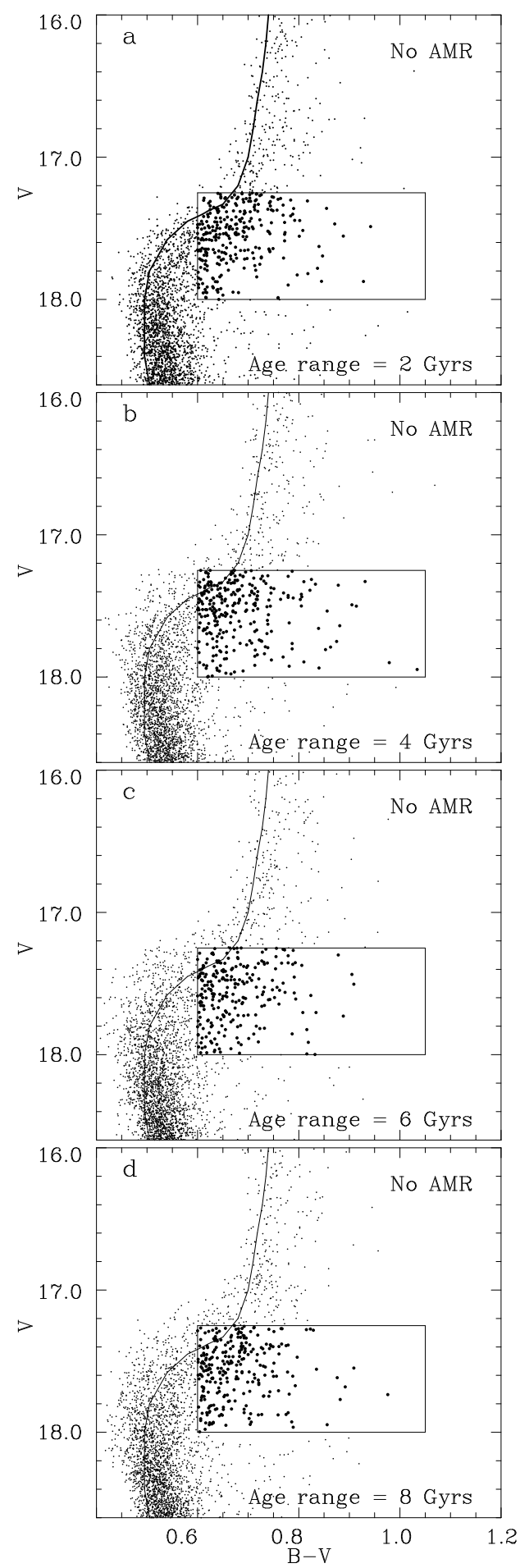

Fig. 20.- Examples of synthetic CMDs obtained from four simulations. Although the simulations have an age range, they do not have an age-metallicity relation. This means there can be old metal-rich stars as well as young metal-poor stars in the simulation. See text $(\S 4.4)$ for details. 